\title{
Electrophilic Alkynylation: The Dark Side of Acetylene Chemistry
}

\author{
Jonathan P. Brand and Jérôme Waser* \\ Received (in $X X X, X X X)$ Xth $X X X X X X X X X 20 X X$, Accepted Xth $X X X X X X X X X 20 X X$ \\ DOI: 10.1039/b000000x
}

${ }_{5}$ In addition to the well-established nucleophilic alkynylation, the use of electrophilic alkynes can expand tremendously the scope of acetylene transfer reactions. The use of metal catalysis has recently led to a rebirth of this research area. Halogenoalkynes, hypervalent alkynyliodoniums, acetylene sulfones and in situ oxidation of terminal acetylenes are the most often used for electrophilic alkynylation. Heteroatoms such as $\mathrm{N}, \mathrm{O}, \mathrm{S}$ and $\mathrm{P}$ can be now efficiently alkynylated. For C-C bond formation, electrophilic 10 acetylenes can be coupled with different organometallics reagents. Recently, the first breakthrough in direct $\mathrm{C}-\mathrm{H}$ and $\mathrm{C}=\mathrm{C}$ bond alkynylation have also been reported. Finally, sulfonyl acetylenes are efficient for alkyne transfer on carbon-centered radicals.

\section{Introduction}

Because of their rigidity, electronic properties and numerous 15 methods available for the functionalization of the triple bond, acetylenes have always been one of the most important functional groups in organic chemistry. Furthermore, alkynes are also important tools and structural elements both in material sciences and chemical biology. ${ }^{1}$ Consequently, it is important to have 20 efficient and flexible methods to introduce a triple bond on all positions of a molecule. Beyond the construction of the triple bond itself, methods allowing the transfer of acetylenes are particularly useful.

If we want to transfer an alkyne, a textbook will tell us to 25 introduce it on an electrophilic position of a molecule. This is due to the high acidity of the $\mathrm{C}-\mathrm{H}$ bond of acetylenes originating from its sp hybridization, which allows the easy formation of numerous nucleophilic acetylide reagents via deprotonation. Two classic examples are the addition of alkynes on ketones or aldehydes to 30 give alcohols ${ }^{2}$ and the Sonogashira coupling of aryl halides and acetylenes (Scheme 1, A). ${ }^{3}$ The reverse approach, the electrophilic alkynylation of the nucleophilic $\alpha$-position of a carbonyl group or an electron-rich $\mathrm{C}-\mathrm{H}$ or $\mathrm{C}$-metal bond on an aromatic compound is usually not considered (Scheme $1, \mathbf{B}$ ). In 35 this case, the normal reactivity of alkynes would need to be inverted (an Umpolung in its broader sense). This is not an easy task, but it has the potential to make acetylene synthesis much more flexible, which would have an enormous impact on applications.

40 Although the development of electrophilic alkynylation methods has a long history, it is only recently that its potential has been investigated more intensively. Several classes of reagents have emerged as particularly promising (Scheme 1, C):

- Halogenoacetylenes are a logical first choice of reagents.

45 Nevertheless, the sp hybridization of the triple bond confers a low reactivity to these reagents, and it is only with the

development of transition metal-catalyzed reactions that their potential becomes fully apparent. The situation can be consequently compared to that of aromatic halides, but the 50 amount of research done so far is insignificant in comparison.

A Nucleophilic Alkynylation
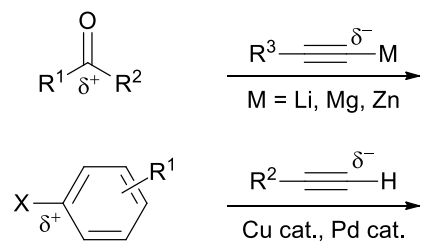

$\mathrm{X}=\mathrm{Cl}, \mathrm{Br}, \mathrm{I}, \mathrm{OR}^{3}$

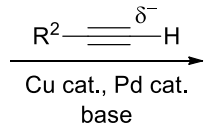

Sonogashira
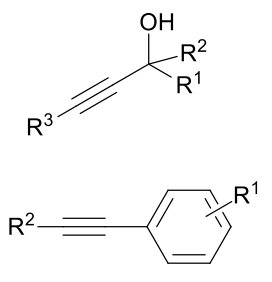

B Electrophilic Alkynylation
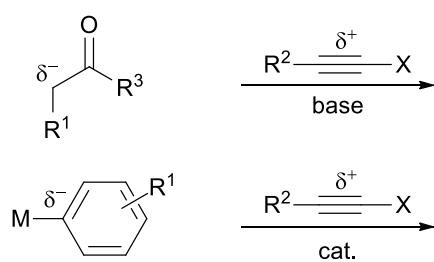

$M=H, M g, Z n, B, \ldots$

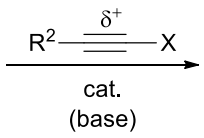

C Electrophylic Alkynylation Reagents
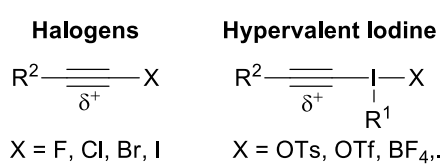

Terminal Alkynes
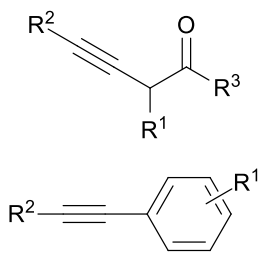
possible with this class of reagents.

- Sulfone-substituted alkynes as electrophilic acetylenes have been less studied, but were found to be demonstrably superior reagents for the alkynylation of nucleophilic radicals.

5 - Finally, in situ oxidation of terminal acetylenes can also be considered to make them electrophilic. This was first investigated using lead(IV) as stoichiometric oxidant. Recently, the use of a metal catalyst together with an external oxidant (ideally dioxygen) has led to more environmentally 10 friendly conditions.

In this tutorial review, we will present selected examples of electrophilic alkynylation classified according to the functionalized nucleophile. ${ }^{5}$ The reactions with inherently nucleophilic heteroatoms will be presented first: in this case, an 15 electrophilic alkynylation method is often the only choice and the resulting electron-rich acetylenes have been recently intensively investigated due to their wide reactivity. In the second part of this review, the focus will turn to the alkynylation of carbon-based nucleophiles including enolates, organometallic nucleophiles, $\mathrm{C}$ ${ }_{20} \mathrm{H} / \mathrm{C}=\mathrm{C}$ bonds and radicals, with a particular emphasis on the choice of electrophilic reagent for a specific reaction. In this context, the use of terminal acetylenes together with an oxidant is sometimes more difficult to classify as electrophilic or nucleophilic. As this specific subject has been recently reviewed, ${ }^{6}$ 25 we will limit ourselves to a few representative examples involving the alkynylation of clearly nucleophilic molecules.

\section{Heteroatom alkynylation}

The presence of a heteroatom substituent on the triple bond has a strong influence on its reactivity. As result of the enhanced 30 nucleophilicity of the triple bond, a range of new chemical transformations can be accessed. Both heteroatoms and alkynes are intrinsically nucleophilic: inverting the polarity of the alkyne is consequently required for the alkynylation of heteroatoms.

\subsection{C-N bond formation}

35 In the two last decades, ynamines and ynamides have become increasingly important in chemical transformations. ${ }^{7,8}$ For example, these compounds can undergo $\alpha$ or $\beta$ addition, oxidation, reduction, cycloaddition or ring-closing metathesis. One of the challenges associated with the use of ynamines and 40 ynamides has been the lack of general methods for their synthesis. In this context, research in this area only became possible with the development of efficient electrophilic alkynylation reactions for amines and amides.

In 1994, Stang and co-workers developed the first synthesis of 45 ynamines using alkynyliodonium salts (Scheme 2). ${ }^{9}$ Lithiated diphenylamine (1) was reacted with an alkynyliodonium triflate to yield the ynamine. It should be noted that this methodology is limited to the synthesis of acetylenes substituted with an electronwithdrawing group (EWG) and diphenylamine to afford push50 pull ynamines.

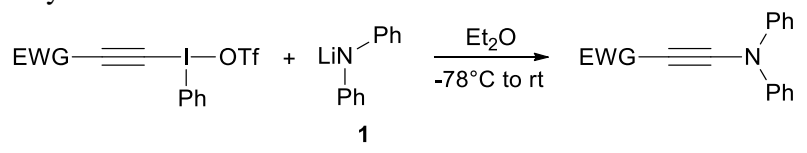

Scheme 2 Ynamine synthesis using alkynyliodonium salts.
The methodology was extended to ynamides by Feldman and 55 co-workers using alkynyliodonium salts having an electronwithdrawing group on the alkyne. ${ }^{10}$ They also reported that alkynyliodonium bearing an alkyl chain are undergoing a 1,5insertion to form a cyclopentene (Scheme 3, A). Witulsky and coworkers then showed that deprotonated sulphonamides and ${ }_{60}$ amides can be alkynylated with trimethylsilylethynyliodonium triflate (2) to form easy to deprotect ynamides (Scheme 3, B). ${ }^{11}$ Based on the results of Feldman and Witulsky, a mechanism involving conjugate addition, iodobenzene $\alpha$-elimination and either 1,2-shift or carbene C-H insertion was proposed (Scheme ${ }_{65} 3, \mathrm{C}$ ). The result of the reaction was linked to the migrating ability of the substituent: proton, silicium and phenyl gave acetylenes, whereas alkyl substituents favoured insertion, limiting the scope of the alkynylation reaction.

A
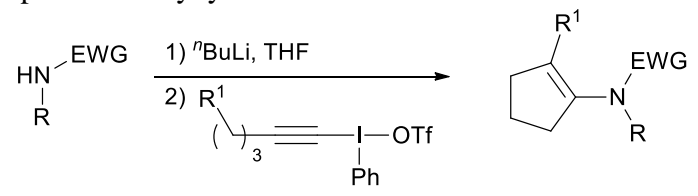

B

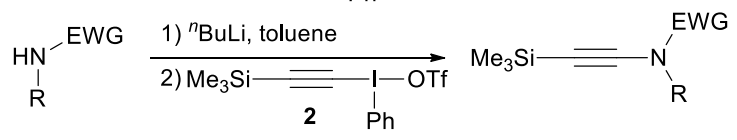

C
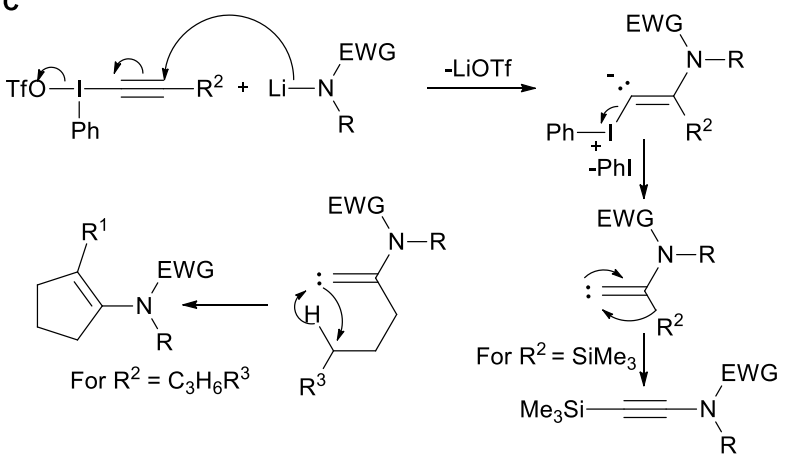

${ }_{70}$ Scheme 3 Ynamide synthesis using alkynyliodonium salts and proposed mechanism.

These limitations were overridden in 2003 when Hsung and co-workers developed the first general copper-catalyzed 75 alkynylation of amides, carbamates and ureas using bromoacetylenes basing on previously reported procedures (Scheme 4). ${ }^{12}$ This breakthrough allowed for the extension of the scope of the acetylene substituent to alkyl groups. The use of bromoacetylenes instead of iodoacetylenes as electrophilic 80 partners was important to significantly reduce the classical homocoupling reaction of acetylenes.

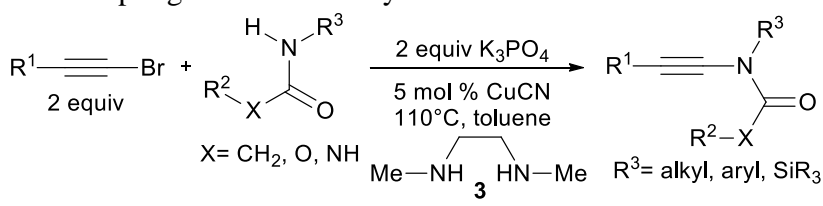

Scheme 4 Copper-catalyzed ynamide synthesis using alkynyl bromides.

85

The same year, Danheiser and co-workers reported a complementary similar method (Scheme 5). ${ }^{13}$ They showed that a stoichiometric preformation of the amide copper species allowed the reaction to proceed at RT. The scope of the reaction could 
also be extended to include sulfonamides and acyclic carbamates. Since these initial works, substantial improvements have been done in the transformation. ${ }^{14}$ Indeed, methods catalytic in copper were developed for the efficient alkynylation of lactams, ureas, 5 carbamates, non-cyclic amides and sulfonamides.

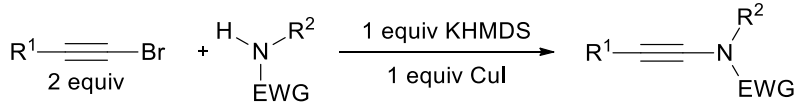

$$
\begin{aligned}
& \text { EWG }=\mathrm{Ts}, \mathrm{CO}_{2} \mathrm{R}, \mathrm{CONHR} \quad \mathrm{R}^{1}, \mathrm{R}^{2}=\text { alkyl, vinyl, aryl, } \mathrm{SiR}_{3}
\end{aligned}
$$

Scheme 5 Ynamide synthesis using alkynyl bromides and a stoichiometric amount of copper salt.

10 In 2008, Stahl and co-workers reported a copper-catalyzed synthesis of ynamides directly from terminal acetylenes (Scheme $6){ }^{15}$ This oxidative coupling has the advantage of avoiding prefunctionalization of the acetylene. A wide range of nitrogen nucleophiles can be used, such as amides, carbamates, ureas, 15 sulfonamides or indoles. A mechanism via sequential ligand exchange of copper anions with the alkyne and the amide followed by a reductive elimination and re-oxidation of the catalyst to $\mathrm{Cu}$ (II) was proposed (Scheme 7).

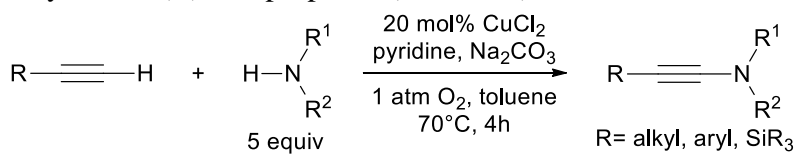

${ }_{20}$ Scheme 6 Copper-catalyzed ynamide synthesis using terminal alkynes.

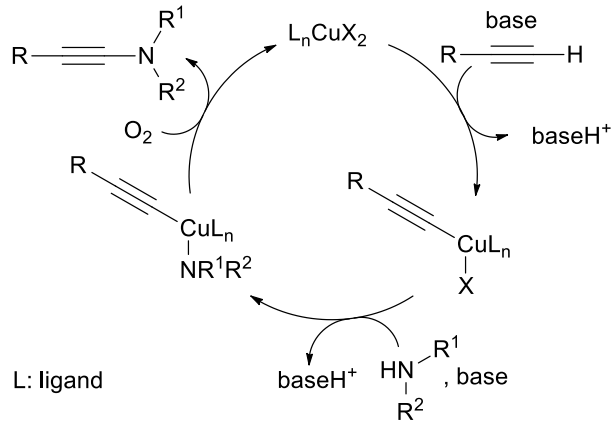

Scheme 7 Proposed mechanism of the copper-catalyzed ynamide synthesis.

25

In addition to halogenoacetylenes and alkynyliodonium salts, further electrophilic acetylenes can be used for particularly challenging $\mathrm{N}$-alkynylations. In 2011, the first synthesis of alkynyl imides was reported by Sueda and co-workers using 30 alkynyl(triaryl)bismuthonium salts and a copper catalyst (Scheme 8). ${ }^{16}$ The mechanism proposed starts with a ligand exchange with the imide. The $\mathrm{Cu}(\mathrm{I})$ species then undergoes an oxidative addition with the alkynyl(triaryl)bismuthonium and then the product is obtained via reductive elimination.

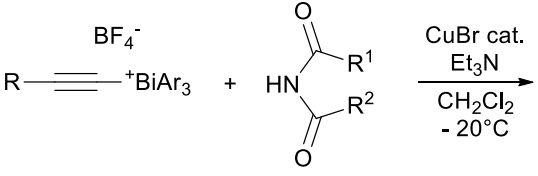

$$
\begin{aligned}
& \mathrm{R}^{1}, \mathrm{R}^{2}=\text { alkyl, aryl }
\end{aligned}
$$

Scheme 8 Copper-catalyzed ynimide alkynyl(triaryl)bismuthonium salts.

\section{${ }_{40}$ 2.2 C-O, C-P and C-S bond formation}

In contrast to ynamides, the reactivity of acetylenes bearing $\mathrm{O}, \mathrm{S}$ or $\mathrm{P}$ substituents have been far less studied up to now despite their high reactivity. With more efficient synthetic methods available, application of these compounds in organic chemistry is 45 also expected to increase in the future.

\section{C-O bond formation}

In the eighties, the development of alkynyliodonium salts lead to 50 the first synthesis of three new classes of compounds: alkynylsulfonates, alkynylcarboxylates and alkynyldialkyl phosphates. ${ }^{17}$ Stang and co-workers discovered that alkynyliodonium tosylates and mesylates are forming alkynyltosylates and alkynylmesylates in the presence of a 55 catalytic amount of copper salt (Scheme 9). ${ }^{18}$ The iodonium species is easily accessed as a stable solid using Koser reagent (hydroxy(tosyloxy)iodobenzene).

$\mathrm{R} \rightleftharpoons \frac{\mathrm{C}_{6} \mathrm{H}_{5} \mathrm{I}(\mathrm{OH}) \mathrm{OTs}}{\mathrm{DCM}, 25^{\circ} \mathrm{C}} \mathrm{R} \rightleftharpoons \underset{\mathrm{Ph}}{\mathrm{I}}-\mathrm{OTs} \frac{\mathrm{CuOTf}}{\mathrm{CH}_{3} \mathrm{CN}, 25^{\circ} \mathrm{C}} \mathrm{R}=$ OTs

${ }_{60}$ Scheme 9 Alkynyltosylate synthesis.

By contrast, the corresponding iodonium carboxylates are much less stable and rearrange to form the corresponding alkynylcarboxylates (Scheme 10). ${ }^{19}$ The method used for the 65 synthesis of the iodonium salt is crucial for good yields. An analogous approach has been used for the formation of alkynyl dialkylphosphates (Scheme 11). ${ }^{20}$ In all these cases, the reaction mechanism involves a classical Michael addition/1,2 shift sequence.

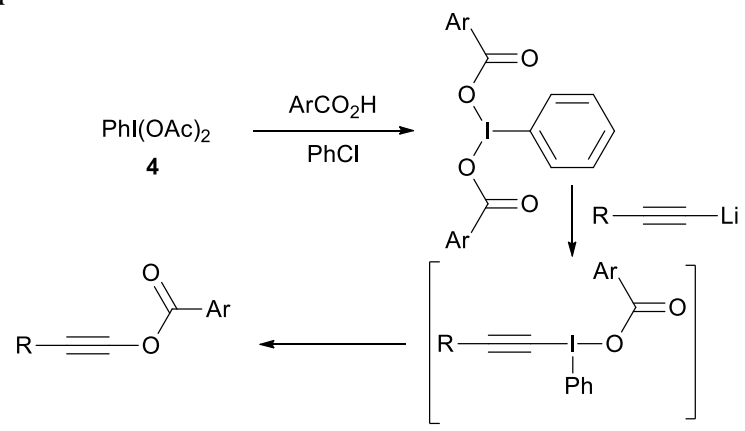

Scheme 10 Alkynylcarboxylate synthesis.
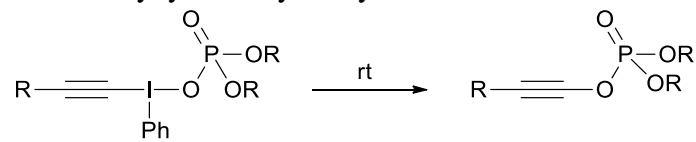

Scheme 11 Alkynylphosphate synthesis.

\section{${ }_{75}$ C-P bond formation}

Alkynylphosphoniums are known to easily undergo Michael addition to form the synthetically useful vinylphosphoniums. Ochiai and co-workers developed the reaction between 80 triphenylphosphine and alkynyliodonium tetrafluoroborates to form alkylethynyl triphenylphosphoniums (Scheme $12, \mathbf{A}){ }^{21}$ Interestingly, this process required light to proceed. On the contrary, the same transformation can be carried out with triflate iodonium salts in the absence of light (Scheme 12, B). ${ }^{22}$ The 
mechanism proposed for the light-mediated reaction is first a photochemical radical transfer to generate I (Scheme 12, C). $\mathrm{PPh}_{3}$ then attacks the iodine center and then intermediate II undergoes a ligand coupling. A radical transfer then generates the 5 product and $\mathbf{I}$. The light-free method was shown to proceed without radical intermediates and is believed to have a classical alkylidenecarbene type mechanism (Scheme 3).
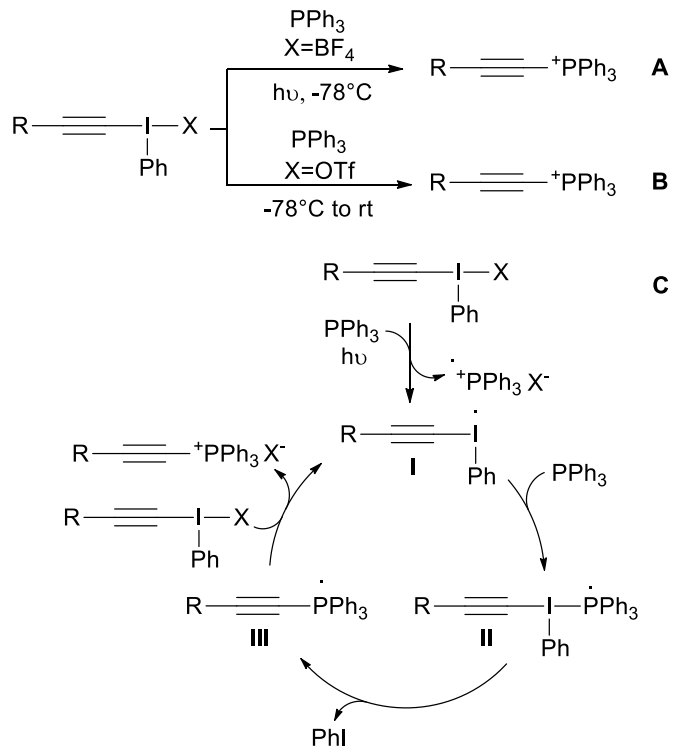

Scheme 12 Alkynylphosphonium syntheses and proposed 10 mechanism.

Recently, a highly atom-economic oxidative coupling between $\mathrm{H}$-phosphonates and terminal acetylenes to form alkynylphosphonates has been developed by Zhao, Han and co15 workers (Scheme 13). ${ }^{23}$ The reaction is analogous to Stahl's method for $\mathrm{N}$ nucleophiles and has a large scope, as well as a large functional group tolerance (including alcohols, amines, cyanide, chloride and amides). Importantly, a nucleoside base analogue was also successfully alkynylated.

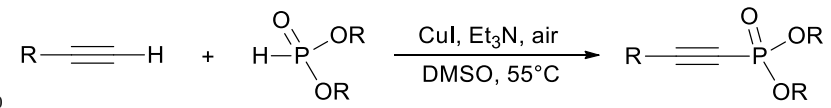

Scheme 13 Alkynylphosphonate synthesis starting from terminal acetylenes.

In 2011, Gaumont and co-workers showed that 25 alkynylphosphines can be obtained efficiently from bromoalkynes and phosphine boranes (Scheme 14). ${ }^{24}$ Phosphine boranes are used in order to avoid the poisoning of the catalyst by the free phosphines.

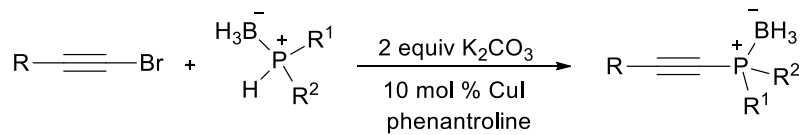

${ }_{30}$ Scheme 14 Alkynylphosphine synthesis using bromoalkynes.

\section{C-S bond formation}

Similarly to nitrogen and oxygen, a range of methods have been 35 developed for the reaction of electrophilic acetylenes with sulfur nucleophiles. One interesting application was reported by Wipf and Venkatraman for the synthesis of thiazoles (Scheme 15). ${ }^{25}$

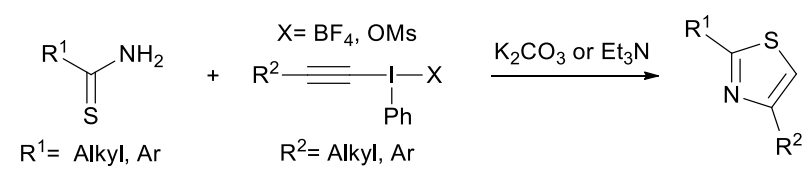

Scheme 15 Regiospecific thiazole synthesis using 40 alkynyliodonium salts.

In 2005, Ochiai and co-workers investigated the mechanism of this reaction (Scheme 16). ${ }^{26}$ Through the isolation of a S-(1alkynyl)thiobenzimidonium salt $\mathbf{5}$, they could demonstrate that 45 the reaction was going through intermediate II. Alkynyl sulfide II is formed from I via a classical conjugate addition on the iodonium reagent followed by a 1,2 shift.
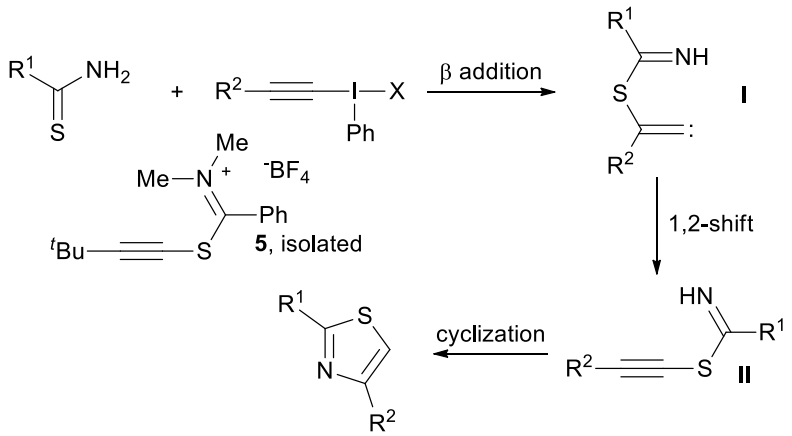

Scheme 16 Mechanism of the thiazole synthesis.

50

In summary, a range of different electrophilic acetylene reagents can be used for heteroatom alkynylation. The choice of the reagent will be guided mainly by the substrate type as well as the substituent tolerated on the alkyne. Recent reports on the use 55 of terminal acetylenes constitute more atom-economic methods. Nevertheless, further development will be required to reach the scope of the preformed electrophilic acetylenes.

\section{Carbon Alkynylation}

\subsection{Enolate Alkynylation}

60

The formation of enolates by deprotonation followed by reaction with electrophiles is one of the most important transformations in organic chemistry. Not surprisingly, the alkynylation of enolates has been investigated for many decades. A seminal result was 65 obtained when Beringer and Galton synthesized the first alkynyliodonium salt and reacted it with the enolate formed from indanedione 6 (Scheme 17). ${ }^{27}$ It was only in 1986, however, that Ochiai and co-workers investigated this reaction in more detail using tetrafluoroborate alkynyliodonium salts (Scheme 18). ${ }^{28,29}$ 70 As with amides (Scheme 3), the product (either 1,2 shift or insertion) depended on the alkyne substituent. The reaction worked especially well for cyclic diketone substrates, but a few examples of the alkynylation of malonates and nitronates were also reported.<smiles>O=C1c2ccccc2C(=O)C1c1ccccc1</smiles>
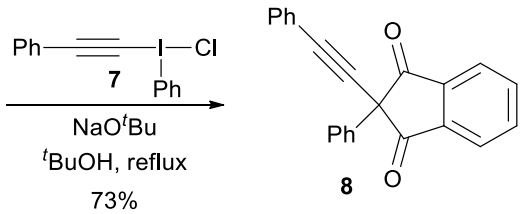

Scheme 17 Diketone $\alpha$-alkynylation using alkynyliodonium salts reported by Beringer. 


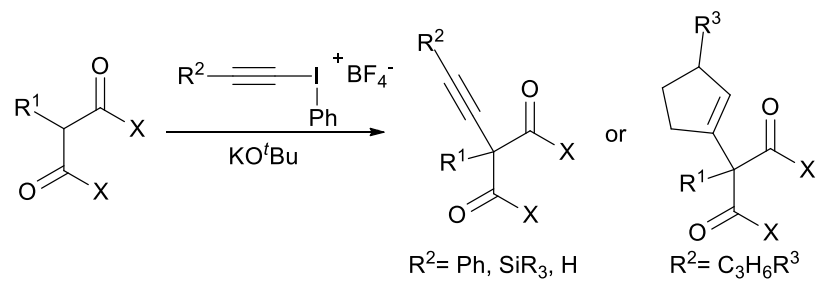

Scheme 18 Diketone $\alpha$-alkynylation using alkynyliodonium salts reported by Ochiai.

An interesting extension of Ochiai's work was proposed in 1991 by Stang and co-workers, who made use of alkynyliodonium triflates for the alkynylation of aminosubstituted malonate 9 (Scheme 19). ${ }^{30}$ This reaction resulted in 10 interesting tertiary propargylic amines and worked even in the case of alkyl-substituted acetylenes. The authors proposed that this was possible due to the good migrating ability of the aminomalonate group itself.<smiles>CCOC(=O)C(N=C(c1ccccc1)c1ccccc1)C(=O)OCC</smiles>
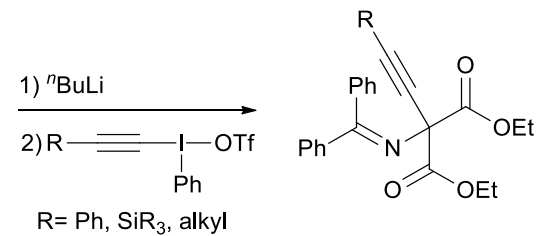

${ }_{15}$ Scheme $19 \alpha$-Aminomalonate alkynylation.

In the 20 years since Stang and Ochiai's work, alkynyliodonium salts were successfully applied several times to the alkynylation of activated enolates, but no further 20 improvement of the methodology has been reported. In 2010, Waser and co-workers discovered the exceptional properties of cyclic Ethynyl-1,2-BenziodoXol-3(1H)-one (EBX) for the alkynylation of activated carbonyl compounds (Scheme 20). ${ }^{31}$

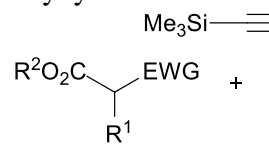

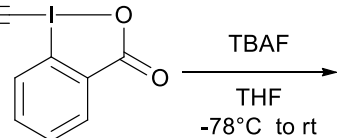

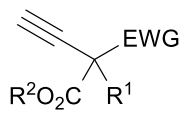

$\mathrm{EWG}=\mathrm{COX}, \mathrm{CN}, \mathrm{NO}_{2}$ $\mathrm{R}^{1}=$ alkyl, aryl<smiles>CC=CCCC(=O)C1C[C+]c2ccccc2C1=O</smiles><smiles></smiles>

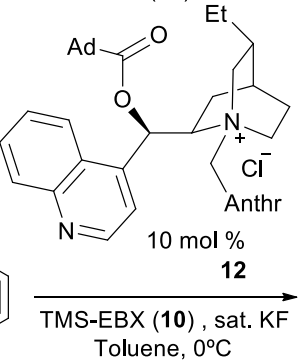

$\mathrm{Ad}=$ adamantyl Anthr= anthracenyl

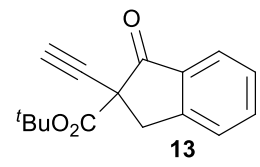

$40 \%$ ee

${ }_{25}$ Scheme 20 Keto-, nitro- and cyano-ester alkynylation using alkynylbenziodoxolone $\mathbf{1 0}$.

EBX was generated in situ from the corresponding silylated reagent $\mathbf{1 0}$ using a fluoride source at low temperature. With this 30 protocol, terminal acetylenes were directly obtained and the generality of the method was significantly increased to include non-cyclic keto-, cyano- and nitro-esters. Furthermore, a first example of asymmetric induction with $40 \%$ enantioselectivity was achieved using a non-soluble fluoride source and a

35 cinchonidine-derived phase transfer catalyst 12. Although the enantioselectivity was still low, it was the first time that asymmetric induction was observed for the alkynylation of enolates using hypervalent iodine reagents.

In 1986, an alternative to hypervalent iodine reagents for the 40 alkynylation of ketoesters and nitro compounds was proposed by Pinhey and co-workers using alkynyl lead derivatives (Scheme 21). ${ }^{32,33}$ The lead reagents are unstable and were generated by transmetallation from the corresponding tin or zinc acetylides. The method is efficient for the alkynylation of acidic $\mathrm{C}-\mathrm{H}$ bonds 45 under mild conditions and has already been applied in the synthesis of more complex molecules. Nevertheless, the requirement for stoichiometric lead and the sensitivity of the formed reagent have so far limited its use.

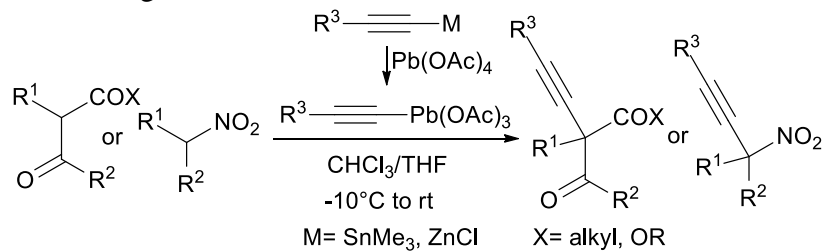

${ }_{50}$ Scheme $21 \beta$-ketoester and nitroalkane $\alpha$-alkynylation using alkynyllead reagents.

Hypervalent iodine and lead reagents were highly successful in the alkynylation of stabilized enolates and nitronates, but they 55 cannot be used for more basic nucleophiles, as the reagents decompose in the presence of strong bases. In the eighties, Kende and co-workers developed the first method for the alkynylation of non-stabilized enolates using chloroacetylenes (Scheme 22). ${ }^{34}$ Bromoacetylenes cannot be used in this reaction, as they act as 60 bromination reagents. The reaction was successful for chloro-, silicium-, sulfur- and aryl- substituted alkynes, but could not be used for aliphatic acetylenes. Kende and co-workers rationalized this result by an addition-elimination mechanism involving an organolithium intermediate II, which cannot be accessed in the 65 case of aliphatic substituents on the alkyne. Dichloroacetylene as a reagent is particularly interesting, as the obtained chloroalkynes can be easily reduced to terminal acetylenes. Importantly, the alkynylation was successful only for the formation of quaternary centers - for monosubstituted enolates, the formation of allenes 70 intermediates was observed, which further react with a second equivalent of nucleophile.

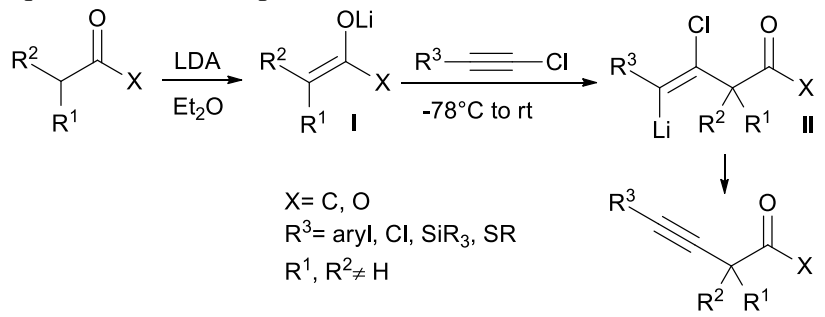

Scheme 22 Ketone $\alpha$-alkynylation using chloroalkynes.

75 In general, halogenoalkynes do not react with stabilized enolates. In order to facilitate Kende's addition-elimination mechanism with these mild nucleophiles, Jørgensen and coworkers introduced further electron-withdrawing groups such as ketones, amides, esters and sulphones onto the acetylene (Scheme $\left.{ }_{80} 23\right){ }^{35}$ With these reagents, the alkynylation became possible 
under mild phase-transfer conditions, which allowed the first highly efficient enantioselective alkynylation of cyclic ketoesters when cinchonidine-derived catalyst 12 was used. Particularly interesting was the use of allyl-substituted propiolates, as the 5 obtained esters can be easily decarboxylated using a palladium catalyst. To the best of our knowledge, Jørgensen's report remains the only highly enantioselective electrophilic alkynylation reported so far.

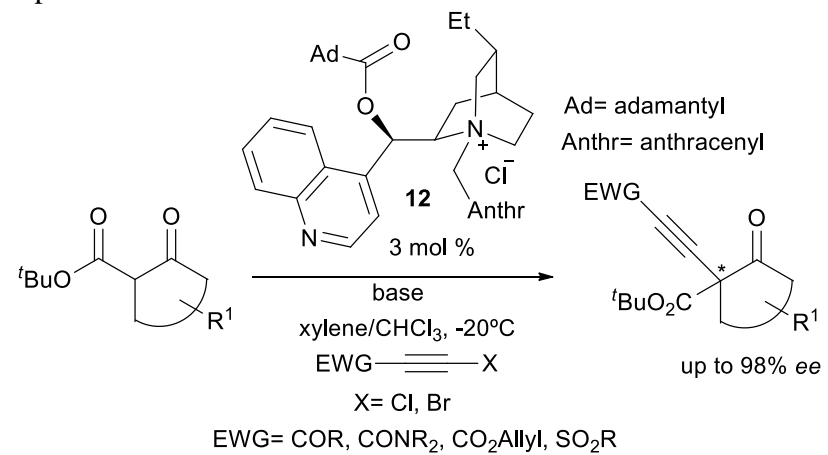

${ }_{10}$ Scheme $23 \beta$-ketoester asymmetric $\alpha$-alkynylation using chloro and bromoalkynes.

The only metal-catalyzed $\alpha$-alkynylation of carbonyls starting from silyl enol ethers was reported by Yamaguchi and co15 workers in 2003 using $\mathrm{GaCl}_{3}$ as a catalyst and chlorosilylacetylene 14 as reagent (Scheme 24). ${ }^{36}$

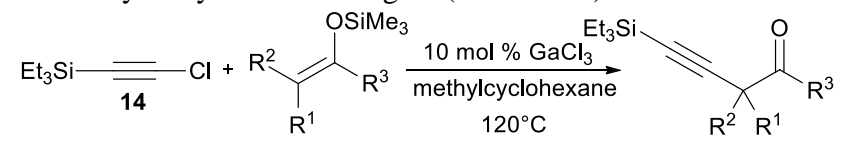

Scheme 24 Silyl enol ether alkynylation using chloroalkyne 14.

20 In 2006, the Yamaguchi group modified this method using sub-stoichiometric amounts of $\mathrm{GaEt}_{3}$ to obtain what remains the only example of direct $\alpha$-alkynylation of ketones (Scheme 25). ${ }^{37}$ The reaction likely proceeded via formation of a gallium enolate followed by insertion and $\beta$-chloride elimination (Scheme 26). In 25 the case of $\mathrm{GaEt}_{3}$, the organometallic reagent also served as a base to generate the enolate, which explained the higher loading required.

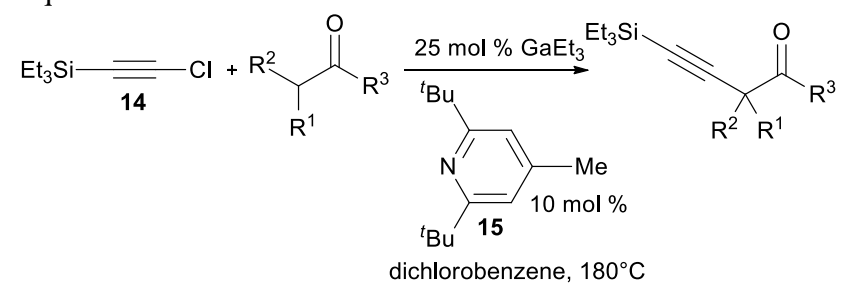

Scheme 25 Ketone $\alpha$-alkynylation using chloroalkynes 14 .

30 To summarize this section, important progress has been realized for the alkynylation of stabilized enolates, including the development of the first enantioselective methods. The alkynylation of non-activated carbonyl compounds remains more limited, and no enantioselective method has yet been reported. 35 Many other important challenges still remain unsolved: the alkynylation to give aliphatic acetylenes and the formation of tertiary propargylic positions are most preeminent among them.

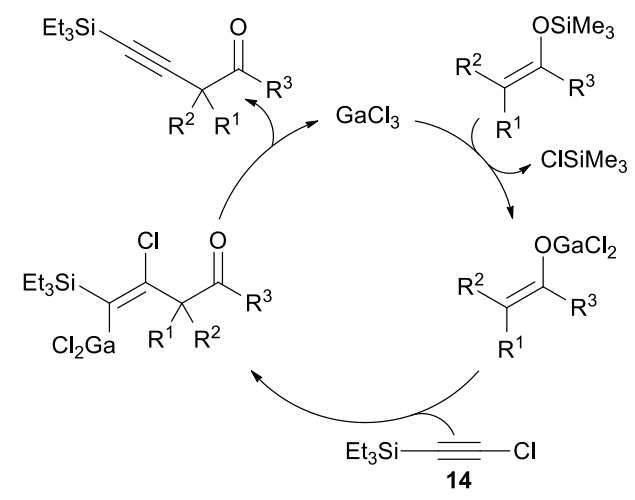

${ }_{40}$ Scheme 26 Mechanism of the $\alpha$-alkynylation.

\subsection{Alkynylation of organometallic nucleophiles}

The alkynylation of organometallic reagents has been demonstrated for many years, but there are only few truly general 45 methods. Analogous to the field of aryl halides, cross-coupling reactions have been especially successful. Nevertheless, the potential of the approach is still far from being fully realized.

\subsubsection{Stoichiometric reactions}

50 The direct alkynylation of hard organometallic reagents, such as Grignard and organolithiums, is usually difficult as numerous side reactions can occur. Interesting pioneering exceptions are the methods reported by Smorada and Truce using alkynyl sulfones (Scheme 27) ${ }^{38}$ and by Sauvêtre and Normant using ethynyl 55 fluoride (16) (Scheme 28). ${ }^{39}$ The yields with sulfones were good, but a hydrogen in propargylic position was not tolerated due to the basicity of organolithium and magnesium reagents.

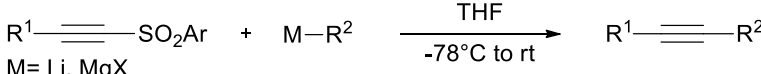

$$
\begin{aligned}
& \mathrm{R}^{1}, \mathrm{R}^{2}=\text { aryl, tertiary alkyl }
\end{aligned}
$$

Scheme 27 Organomagnesium and organolithium alkynylation 60 using alkynylsulfones.

In contrast, the alkynylation using ethynyl fluoride (16) displayed an impressive scope, including aryl, heteroaryl and primary, secondary and tertiary organolithium and Grignard 65 reagents (Scheme 28). However, two equivalents of an organometallic reagent are required, due to the presence of the acidic $\mathrm{C}-\mathrm{H}$ bond. On the other hand, the obtained acetylide can be quenched by a variety of electrophiles, such as proton (water), aldehydes, chlorosilanes or aliphatic bromides. Unfortunately, 70 ethynyl fluoride (16) is a hazardous reagent, which needs to be generated in situ from difluoroethene at very low temperature. This limitation probably explains why the method has not found a widespread use in organic chemistry.

$$
\begin{aligned}
& \mathrm{R}^{1}-\mathrm{M}+\mathrm{H}-\overline{\overline{\mathbf{1 6}}} \mathrm{F} \\
& 2 \text { equiv. } \\
& \mathrm{M}=\mathrm{Li}, \mathrm{MgY} \\
& \mathrm{R}^{1}=\text { (hetero)aryl, alkyl }
\end{aligned}
$$

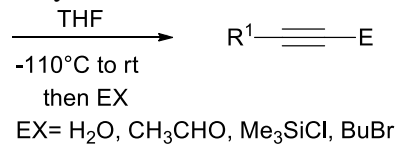

${ }_{75}$ Scheme 28 Organomagnesium and organolithium alkynylation using fluoroacetylene $\mathbf{1 6}$.

In principle, the use of less basic organometallic reagents should allow the alkynylation of a broader scope of substrates. In 80 this respect, organocopper reagents have been especially successful. In addition to the lower basicity of the carbon ligand, 
copper is also a redox active metal, which opens new pathways for the reaction via oxidative addition/reductive elimination. In 1987, Stang and Kitamura made use of the exceptional reactivity of alkynyliodonium tosylates for the alkynylation of 5 alkenylcopper reagents, which were themselves easily obtained via syn-cupration of terminal alkynes with alkylcopper reagents (Scheme 29). ${ }^{40}$ This method allowed an efficient stereoselective synthesis of enynes.

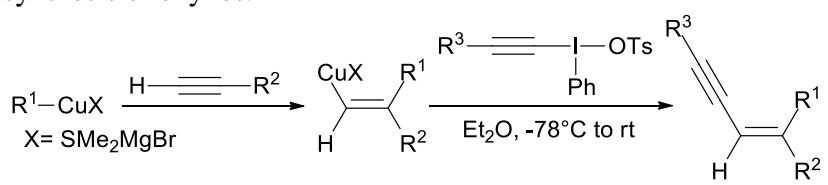

${ }_{10}$ Scheme 29 Alkenyl organocopper reagent alkynylation using alkynyliodonium salts.

For the synthesis of aliphatic acetylenes, Yeh and Knochel developed a very efficient method based on a mixed $\mathrm{Zn}-\mathrm{Cu}$ 15 reagent (Scheme 30). ${ }^{41}$ Treatment of an organozinc with $\mathrm{CuCN} \cdot \mathrm{LiCl}$ followed by reaction with an iodo or bromo acetylene lead to aliphatic alkynes in high yield.

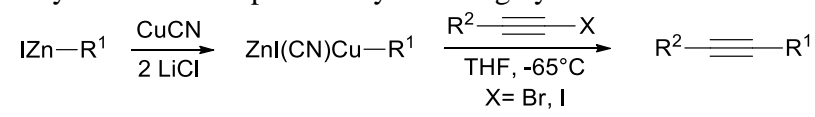

Scheme 30 Organocopper reagent alkynylation using bromo and 20 iodo alkynes.

Due to the very mild nature of the organocopper/zinc reagent, this reaction is tolerated by many functional groups. It has been used successfully in many applications by the Knochel group, but 25 also by other researchers. For example, Hupe and Knochel reported an asymmetric hydroboration/transmetallation /alkynylation protocol for the enantioselective hydroalkynylation of olefins (Scheme 31). ${ }^{42}$ In this case, the acetal in the product can be easily hydrolysed to give the corresponding aldehyde.

${ }_{30}$ Conceptually, this transformation is very interesting, as it is the equivalent of the electrophilic alkynylation in $\beta$ position of a carbonyl group and consequently constitutes a double Umpolung of the reactivity.<smiles>C1=C(C2OCCO2)CCCC1</smiles>

17

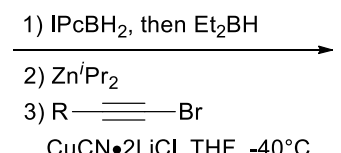

formally:<smiles>[R]C#[13C]CC1CC[CH+]CC1</smiles>

${ }_{35}$ Scheme 31 Formal $\beta$-alkynylation of aldehydes using bromoalkynes.

In 2010, Baran and co-workers used Knochel's protocol in the total synthesis of the dimeric tryptamine alkaloid psychotrimine ${ }_{40}$ (Scheme 32) ${ }^{43}$ In this reaction, the alkynylation of $\mathbf{1 8}$ was successful in the presence of both an ester and a free $\mathrm{NH}$ bond. The introduced alkyne could then be used in a Larock indole synthesis to access the natural product. These two examples among others illustrate the power of the Knochel's approach, 45 which is still one of the most often used methods for the

alkynylation of aliphatic nucleophiles.

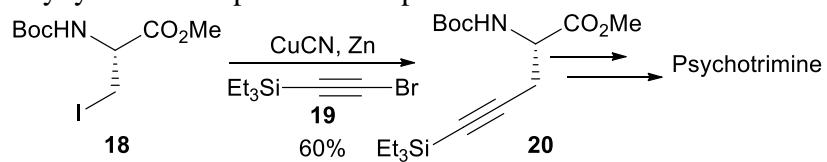

Scheme 32 Organocopper reagent alkynylation in the synthesis of psychotrimine.

50

\subsubsection{Catalytic Reactions}

Another solution to make the electrophilic alkynylation of organometallic reagents more efficient is the use of a transition metal catalyst, especially palladium, but also nickel or copper.

${ }_{55}$ The most probable catalytic cycle of such reactions is very similar to the classic Sonogashira cross-coupling, except that the role of nucleophile and electrophile is inverted (Scheme 33). So far, both bromo and iodo acetylenes as well as hypervalent iodine reagents have been used. Despite several examples, cross60 coupling involving aryl nucleophiles ((Scheme 33, A) has been used only rarely in organic synthesis. The use of alkenyl nucleophiles (Scheme 33, B) has been more commonly documented, in particular in the context of the synthesis of enynes natural products. This is due to the fact that vinyl 65 organometallic reagents are easily obtained from the hydro- or carbo-metallation of the corresponding alkynes. In particular, Negishi utilized aluminium or zirconium reagents as nucleophiles in the presence of a catalytic amount of zinc, ${ }^{44}$ and Suzuki used sialyl boranes ${ }^{45}$ for the highly stereoselective synthesis of enynes. 70 The most atom-economic approach, the Heck reaction between olefins and electrophilic alkynes has been only rarely examined, both with alkynyl bromides ${ }^{46}$ and alkynyliodonium salts. ${ }^{47}$

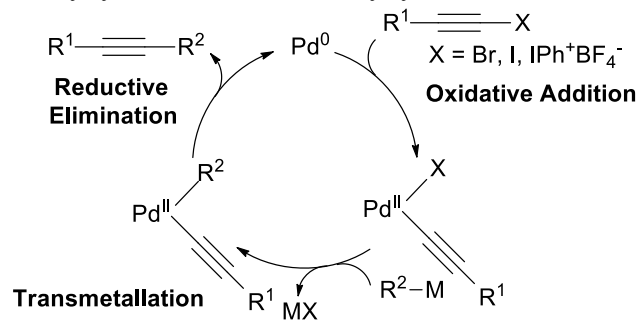

$$
\mathrm{R}^{2}-\mathrm{M}=\mathrm{A} \quad \mathrm{Ar}-\mathrm{M} \quad(\mathrm{M}=\mathrm{B}, \mathrm{Sn}, \mathrm{Zn}, \ldots)
$$

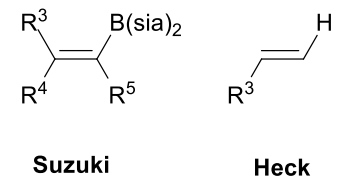

Scheme 33 Palladium-catalyzed alkynylation of organometallic 75 reagents.

Finally, aliphatic organometallic reagents constitute a more challenging class of nucleophiles for cross-coupling reactions. Furthermore, the Sonogashira reaction is less efficient with 80 aliphatic halides as electrophiles. Nevertheless, stoichiometric reactions (see section 3.2.1) have long been the method of choice for the synthesis of alkyl acetylenes. It was only in 2010 that Cahiez and co-workers reported a very general copper-catalyzed cross-coupling of Grignard reagents with alkynyl halides ${ }_{85}$ (Scheme 34). ${ }^{48}$ The method worked for both alkyl and aryl Grignard reagents and acetylenes. A bromo substituent was 
optimal for the transfer of alkyl acetylenes, whereas chloro was better for phenyl alkynes. The mild reaction conditions resulted in a high tolerance towards functional groups such as ethers, amines or esters.

$$
\begin{aligned}
& \mathrm{R}^{1}=\mathrm{X}+\mathrm{XMg}-\mathrm{R}^{2} \quad \frac{\mathrm{CuCl} \text { cat. }}{\mathrm{NMP}, \mathrm{THF}, 0^{\circ} \mathrm{C}} \quad \mathrm{R}^{1}=\mathrm{R}^{2} \\
& \mathrm{R}^{1}=\text { alkyl, aryl } \quad \mathrm{R}^{2}=\text { alkyl, aryl } \\
& \mathrm{X}=\mathrm{Br}, \mathrm{Cl}
\end{aligned}
$$

Scheme 34 Copper-catalyzed alkynylation of Grignard reagents using bromo and chloroalkynes.

The reaction was proposed to proceed not via a classical 10 oxidative addition/reductive elimination mechanism, but via formation of a cuprate, followed by cupration of the triple bond and $\beta$-halogen elimination (Scheme 35). In fact, this type of catalytic cycle involving no change of oxidation state at the metal can also be proposed for palladium catalysis.

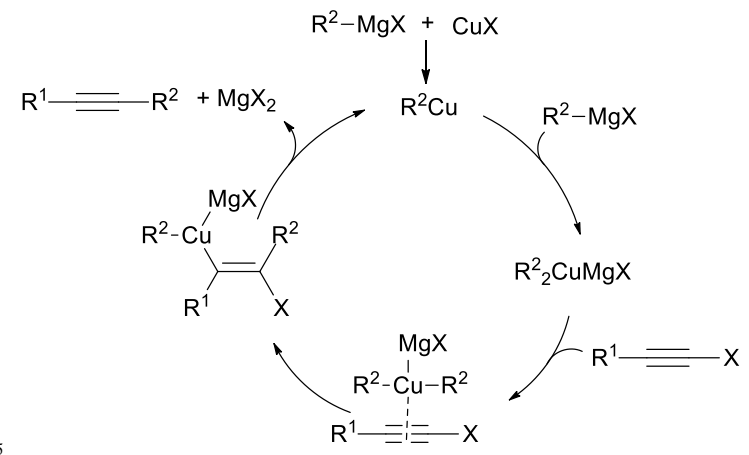

Scheme 35 Mechanism of the copper-catalyzed alkynylation.

If non-symmetrical alkyl reagents are used, the stereoselectivity of the reaction becomes important. This issue 20 has been addressed only very recently by Knochel and coworkers for the alkynylation of cyclohexyl zinc reagents (Scheme 36). ${ }^{49}$ Starting from a diastereomeric mixture of organozincs, a single diastereoisomer was obtained using a bathocupreinpalladium catalyst. A possible explanation for the observed high 25 diastereoselectivity is the formation of the thermodynamically more stable palladium intermediate, followed by stereoretentive reductive elimination.

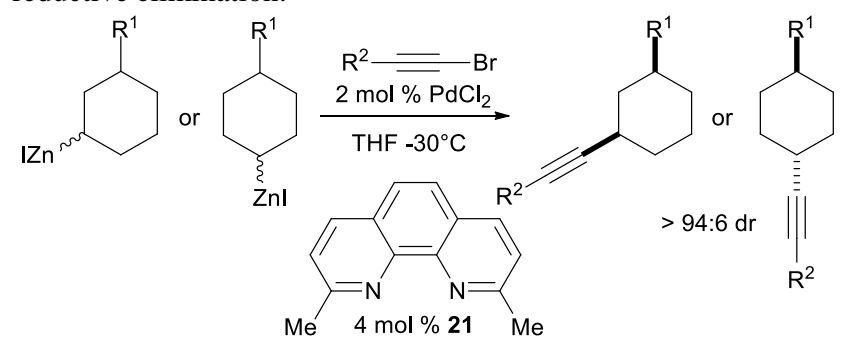

${ }_{30}$ Scheme 36 Palladium-catalyzed alkynylation of organozinc reagents using bromoalkynes.

To summarize this section, although there are numerous examples of alkynylation of organometallic reagents- both with 35 and without catalyst- there are only a few truly general methods that are routinely used in organic synthesis. Nevertheless, the recent impressive results of the groups of Cahiez and Knochel revealed a huge neglected potential, especially when considering that the area of stereoselective (both enantioselective and 40 diastereoselective) reactions is nearly untouched.

\subsection{Alkynylation of $\mathrm{C}-\mathrm{H}$ and $\mathrm{C}=\mathrm{C}$ bonds}

In the previous section, we presented the alkynylation of organometallic reagents. This approach requires modification of 45 terminal acetylenes and often generation of organometallic reagents from the corresponding halides. Halides and terminal acetylenes are the traditional partners of the Sonogashira reaction, so that the extra steps required for the "inverse reactivity" approach are difficult to justify. In contrast, if electrophilic 50 alkynylation of $\mathrm{C}-\mathrm{H}$ and $\mathrm{C}=\mathrm{C}$ bonds can be achieved directly, the transformation becomes much more interesting from the point of view of synthetic efficiency. With the exception of the venerable Cadiot-Chodkiewicz reaction for the alkynylation of acetylenes, this exciting area of research has emerged only very recently, 55 with most examples being reported after 2002.

\subsubsection{Alkynyl C-H bonds: the Cadiot-Chodkiewicz reaction}

Diynes are very important building blocks in organic synthesis. They are present in natural products and in electronic and optical 60 organic materials. They can also be used for the synthesis of heterocycles, in particularly pyrroles and thiophenes. Despite some recent progress in the direct cross-coupling of terminal acetylenes, ${ }^{6}$ the copper-mediated coupling of terminal acetylenes and alkynyl halides discovered by Cadiot and Chodkiewicz in 651955 still remains the method of choice for the synthesis of nonsymmetric diynes (Scheme 37). ${ }^{50,51}$ Bromoalkynes are usually the reagent of choice, although iodoalkynes have also been used. Chloroalkynes are usually not reactive enough.

$$
\mathrm{R}^{1}=\mathrm{Br}+\mathrm{H} \rightleftharpoons \mathrm{R}^{2} \underset{\mathrm{EtNH}_{2}}{\stackrel{\mathrm{CuCl}}{\rightleftharpoons}} \mathrm{R}^{1}=\mathrm{R}^{2}
$$

${ }_{70}$ Scheme 37 Cadiot-Chodkiewicz alkynylation of terminal alkynes.

In fact, this transformation constitutes the only name reaction based on electrophilic alkynylation, and it has already been used 75 more than 1000 times in organic synthesis. It is clear that a full coverage of this reaction goes far beyond the scope of this review. A recent impressive example is given in Scheme 38 to provide a taste of the generality and functional group tolerance of the reaction: in the synthesis of (-)-gummiferol, Takamura and 80 co-workers reported the successful Cadiot-Chodkiewicz coupling of 22 with alkynyl bromide $\mathbf{2 3}$, a substrate containing both a vinylic and a propargylic epoxide, as well as a free allylic alcohol (Scheme 38). ${ }^{52}$ Only minor changes have been made to the original protocol, the most important being the use of 85 hydroxylamines or hydrazines as co-reductant to keep the catalyst in the active $\mathrm{Cu}(\mathrm{I})$ oxidation state. In certain cases, the use of silyl, stannyl, Grignard or organozinc acetylenes with halogenoalkynes has also been reported using different metal catalysts. Recently, Lei and co-workers ${ }^{53}$ and Frauenrath and co90 workers $^{54}$ reported that palladium could sometimes be a superior catalyst with terminal acetylenes and alkynyl zinc respectively. 


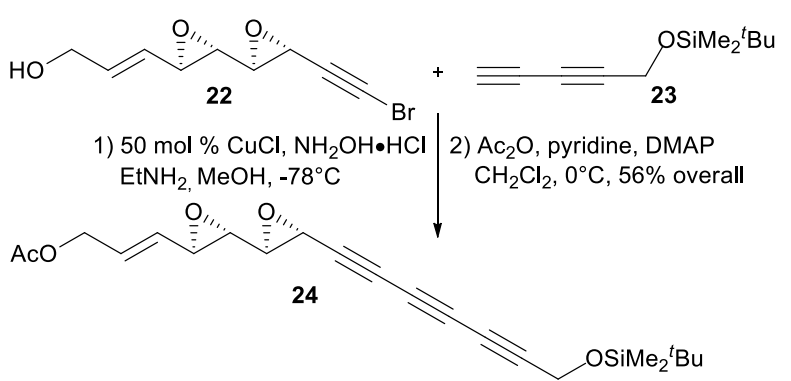

Scheme 38 Application of the Cadiot-Chodkiewicz reaction in total synthesis.

\section{3.3.2 Aromatic C-H bonds}

In contrast to arylation and vinylation of aromatic $\mathrm{C}-\mathrm{H}$ bonds, alkynylation has been far less studied up to 2009. This field has been advanced by Yamaguchi and co-workers, who showed that chlorosilylethyne 14 and catalytic $\mathrm{GaCl}_{3}$ allowed the ortho10 alkynylation of phenols (Scheme 39). ${ }^{55}$ The use of a silylated electrophilic acetylene was key for the development of catalytic conditions. In addition, chloroacetylenes were far better than the corresponding iodo and bromo acetylenes. Phenyl(trimethylsilylethynyl)iodonium $p$-toluenesulfonate did not yield any 15 product. A pyridine additive was added to avoid competitive desilylation.

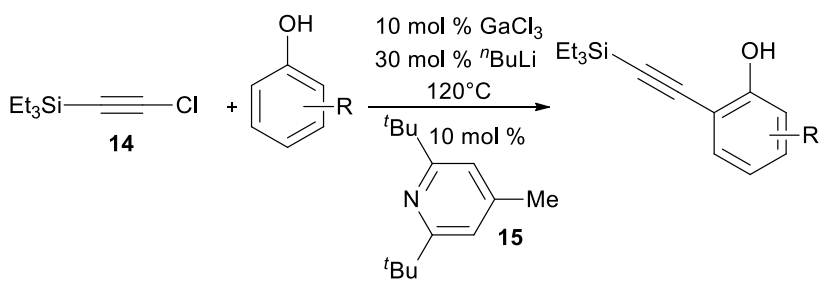

Scheme 39 Gallium-catalyzed ortho-alkynylation of phenols using chloroalkynes.

20

The reaction was proposed to proceed via formation of a gallium phenolate (Scheme 40). The next step is a directed carbogalliation. A $\beta$-chloro elimination then affords the product and regenerates the catalyst. This mechanism is very similar to 25 the one proposed for enolates (Scheme 26).

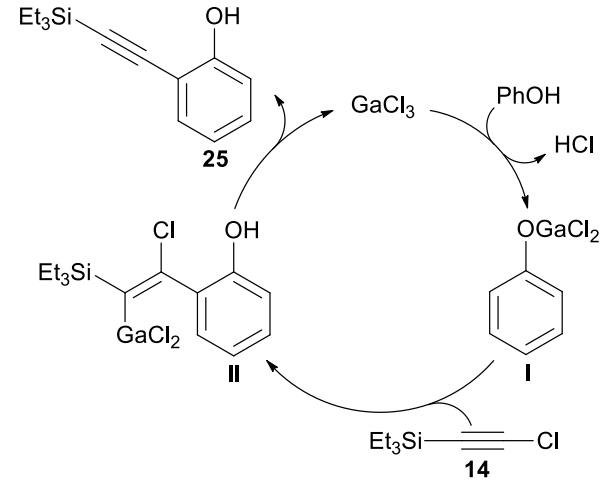

Scheme 40 Mechanism of the gallium-catalyzed orthoalkynylation of phenols using chloroalkyne $\mathbf{1 4}$.

30 An analogous method was later developed for the directed ortho-alkynylation of anilines by Yamaguchi and co-workers (Scheme 41) ${ }^{56}$ Both alkyl and aryl substituents were tolerated on the nitrogen atom while electron-donating groups and halogens were tolerated on the aromatic ring. The method afforded alpha35 alkynyl anilines, which are known precursors to indoles.

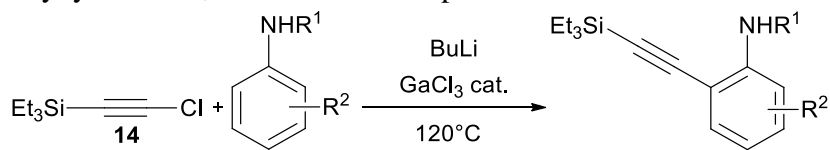

Scheme 41 Gallium-catalyzed ortho-alkynylation of anilines using chloroalkyne 14.

40 In 2004, Trofimov and co-workers reported the first direct alkynylation of pyrroles and introduced the terminology of inverse Sonogashira coupling (Scheme 42). ${ }^{57}$ The scope of alkynes was limited to bromoacetylenes bearing an electronwithdrawing group. The reaction was solvent-free and occurred 45 in the presence of a 10 fold mass excess of $\mathrm{Al}_{2} \mathrm{O}_{3}$. The most probable mechanism involves nucleophilic conjugate addition followed by elimination.

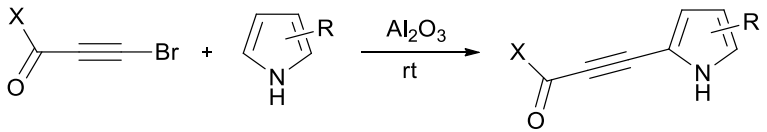

Scheme 42 Pyrrole alkynylation of pyrroles using bromoalkynes. 50

In 2007, Gevorgyan and co-workers developed the first example of transition metal-catalyzed alkynylation of aromatic C$\mathrm{H}$ bonds (Scheme 43). ${ }^{58}$ In this work, they showed that bromoalkynes could be used for the alkynylation of $\mathrm{N}$-fused 55 heterocycles. Importantly, acetylenes substituted with aromatics, aliphatic and trialkylsilyl groups all resulted in the alkynylation product. In contrast, both iodo and chloroalkynes gave poor results. As an analogy to the arylation of electron-rich heterocycles, the reaction was proposed to proceed via $\mathrm{Pd}^{0} / \mathrm{Pd}^{\mathrm{II}}$ 60 cycle involving oxidative addition of $\mathrm{Pd}^{0}$ on the bromoalkyne (Scheme 44). This highly electrophilic species then undergoes an aromatic electrophilic substitution and the product is finally obtained via a reductive elimination.

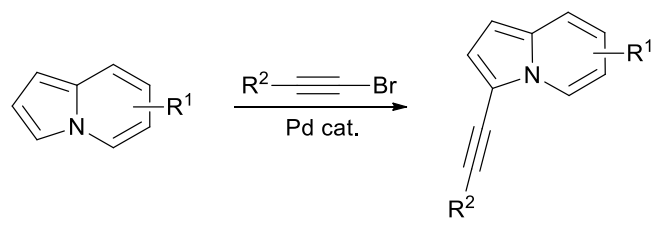

${ }_{65}$ Scheme 43 Palladium-catalyzed alkynylation of fused Nheterocycles using bromoalkynes.

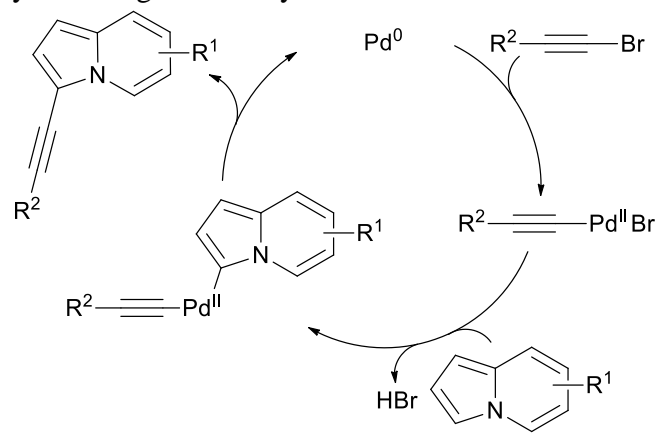

Scheme 44 Mechanism of the palladium-catalyzed alkynylation of fused N-heterocycles.

70

The same concept was used by $\mathrm{Gu}$ and Wang for a $\mathrm{C}_{3}$ regioselective alkynylation of unprotected indoles with 
bromoalkynes (Scheme 45). ${ }^{59}$ The reaction was limited to vinyl and aryl acetylenes.

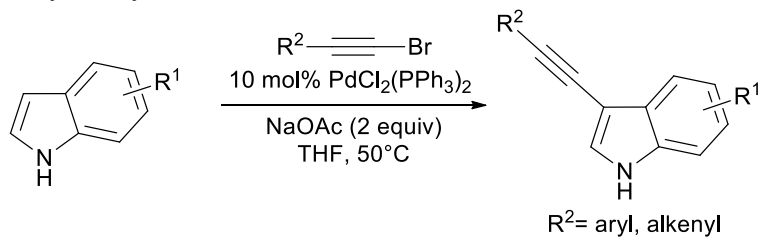

Scheme 45 Palladium-catalyzed alkynylation of indoles using 5 bromoalkynes.

Chatani, Tobisu and co-workers also used palladium catalysis for the ortho alkynylation of anilides (Scheme 46). ${ }^{60}$ The first step was proposed to be a cyclometalation followed by insertion 10 onto the alkynyl bromide which affords II (Scheme 47). A $\beta$ bromide elimination results in the product as well as $\mathrm{PdXBr}$. The use of AgOTf allows regeneration of the active catalyst by removal of the halogen.

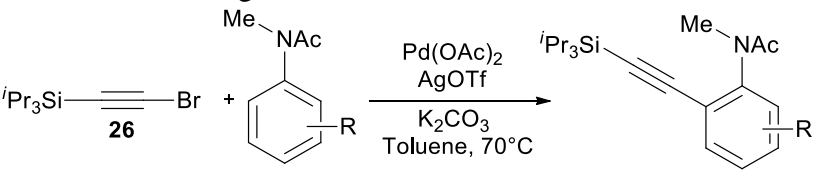

${ }_{15}$ Scheme 46 Palladium-catalyzed ortho-alkynylation of acetamides using bromoalkynes.

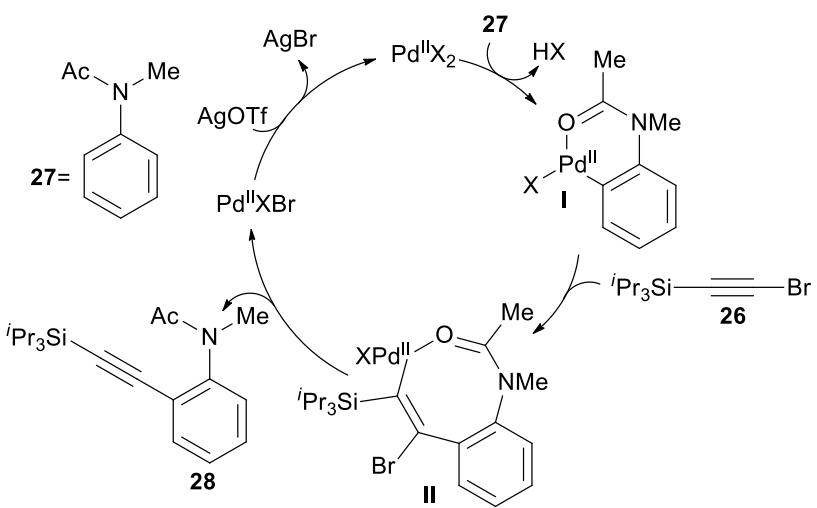

Scheme 47 Mechanism of the palladium-catalyzed ortho20 alkynylation of acetamides.

This methodology was then extended to carboxylic acid derivatives by Chatani, Tobisu and co-workers (Scheme 48). ${ }^{61}$ The use of a bidendate directing group was mandatory. 25 Interestingly, a silver salt was not needed. Both the silyl protecting group and the quinolone directing group can be selectively cleaved.

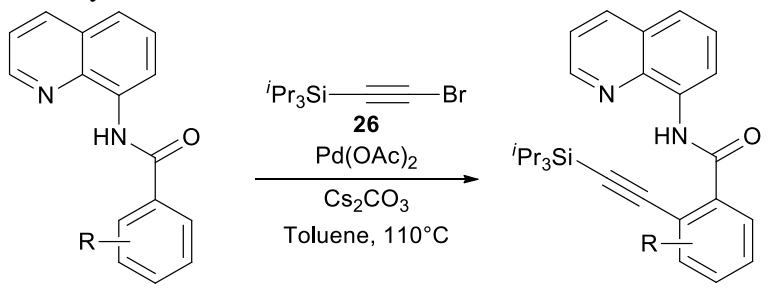

Scheme 48 Palladium-catalyzed ortho-alkynylation of carboxylic 30 acid derivatives using bromoalkynes.

The combination of alkynyl bromides with a transition metal catalyst $(\mathrm{Pd}, \mathrm{Ni}, \mathrm{Cu})$ was also used for the direct alkynylation of azoles in the presence of a base (Scheme 49) ${ }^{62,63,64}$ Deprotonation 35 of the azole increased its reactivity, making it comparable to the one of electron-rich aromatics.

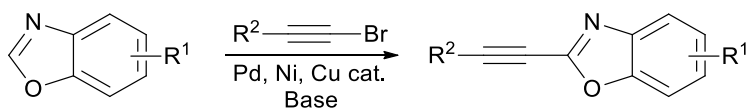

Scheme 49 Catalytic alkynylation of azoles using bromoalkynes.

40 In 2009, Waser and co-workers used for the first time another electrophilic acetylene, namely 1-[(TriIsoPropylSilyl) Ethynyl]1,2-BenziodoXol-3(1H)-one (TIPS-EBX, 29), for the direct alkynylation of indoles and pyrroles (Scheme 50). ${ }^{65}$ Both the triisopropyl group and the benziodoxolone were mandatory for 45 successful alkynylation. The reaction had the regioselectivity of an electrophilic aromatic substitution. A large number of functionalities such as alcohols, ketones, phenols, carboxylic acids, cyano, nitro, iodo and bromo groups were tolerated.

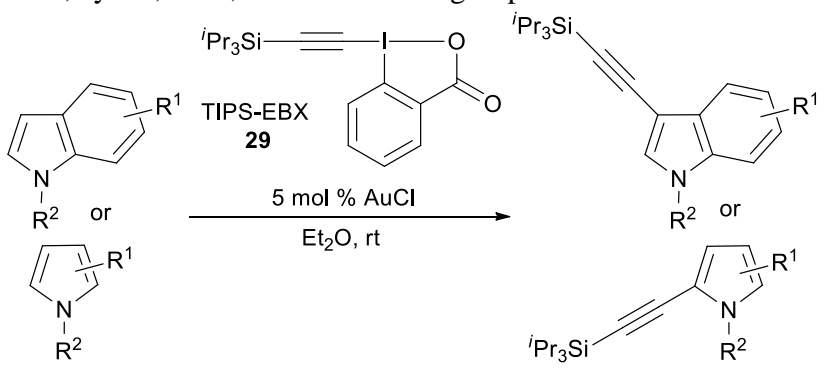

${ }_{50}$ Scheme 50 Gold-catalyzed alkynylation of indoles and pyrroles using alkynyl benziodoxolone 29.

Two types of mechanisms were envisaged: an oxidative and a $\pi$ activation mechanism (A and $\mathbf{B}$ in Scheme 51).

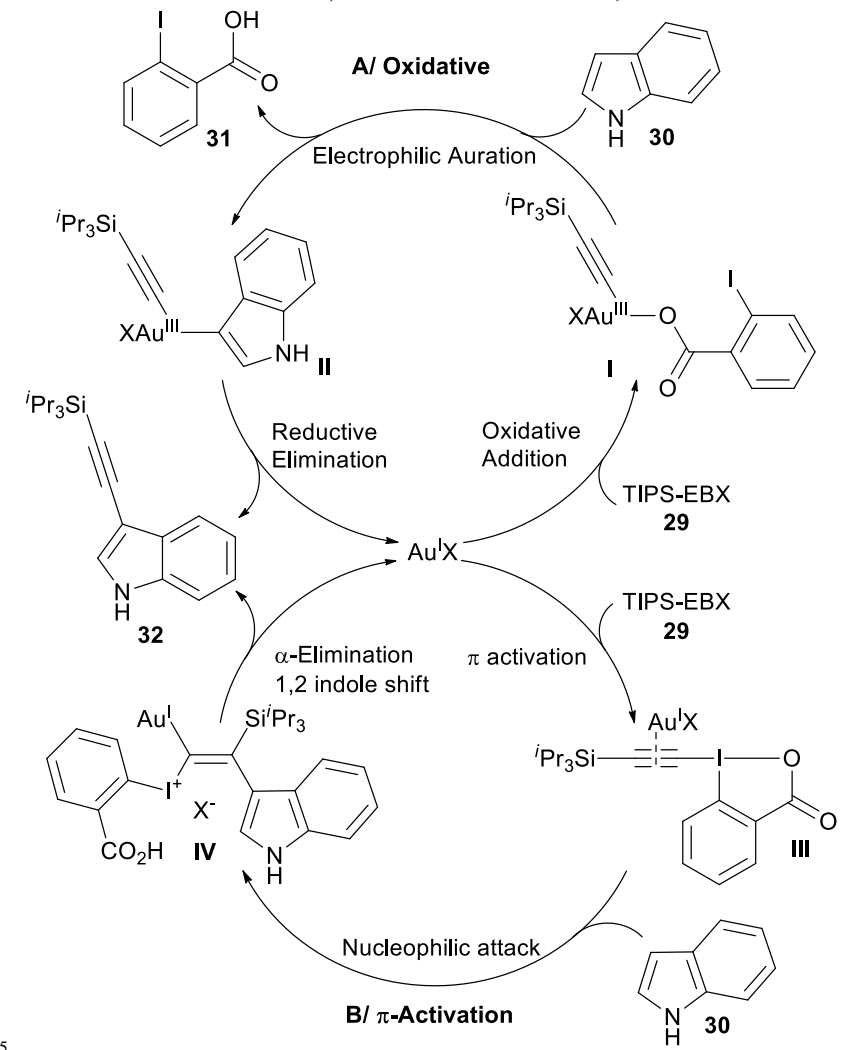

Scheme 51 Possible mechanisms of the gold-catalyzed alkynylation of indoles and pyrroles. 
Recently the methodology was extended to anilines and trimethoxybenzenes by Brand and Waser (Scheme 52). ${ }^{66}$ As the reaction did not rely on a directing group effect, an unprecedented para selectivity was obtained.

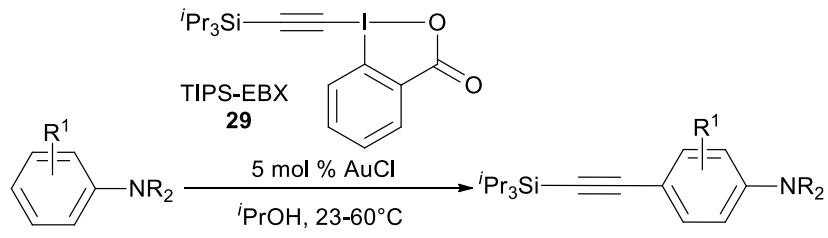

$\mathrm{R}=\mathrm{alkyl}$

Scheme 52 Gold-catalyzed para-alkynylation of anilines using alkynyl benziodoxolone 29 .

Alkynylthiophenes are finding many applications in organic 10 materials as extended $\pi$ conjugated systems. Thiophenes alkynylation is especially challenging due to their low nucleophilicity compared to indoles and pyrroles. As a result, the Au-catalyzed methodology could not be applied to thiophenes. Nevertheless, Brand and Waser discovered that the addition of 15 Brønsted acid led to a cooperative activation of TIPS-EBX (29) and thus allowed the direct alkynylation of thiophenes (Scheme 53). ${ }^{67} \mathrm{~A}$ range of building blocks relevant to materials science, such as 2-hexylbithiophene and 3,4-ethylenedioxythiophene (EDOT) were successfully alkynylated.

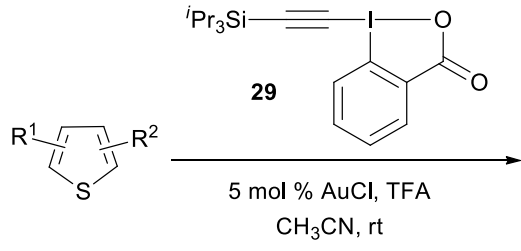

20

Scheme 53 Cooperative gold/Brønsted acid activation of 29 for the alkynylation of thiophenes.

Since 2010, several methodologies employing directly terminal 25 acetylenes have also been reported. ${ }^{6}$ Nevado and de Haro developed the gold-catalyzed alkynylation of methoxybenzenes, $\mathrm{N}$-benzylindole and N-benzylpyrrole using triphenylphosphine gold chloride and phenyl iododiacetate (PIDA) (Scheme 54). ${ }^{68}$ Best results were obtained with acetylenes bearing electron30 withdrawing groups. Both an oxidative and a $\pi$ activation mechanism were proposed.<smiles>[R10]Oc1cc([R20])cc(O[R1])c1</smiles>

$$
\underset{\substack{\mathrm{PaHCO}_{3} \\ \mathrm{PPh}_{3} \mathrm{AuCl}, \mathrm{Phl}(\mathrm{OAc})_{2}}}{\longrightarrow}
$$

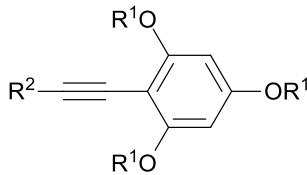

Scheme 54 Gold-catalyzed alkynylation of trimethoxybenzenes using terminal acetylenes.

35

$\mathrm{Su}$ and co-workers and Miura and co-workers reported that aromatic terminal acetylenes could also be used for the alkynylation of polyfluorinated benzenes using copper catalysis (Scheme 55). ${ }^{69,70}$ DDQ was proposed as an electron-transfer 40 mediator. The proposed mechanism is similar to the alkynylation of amides developed by Stahl (Scheme 56). Alternatively, the aromatic group can coordinate to the copper prior to the alkyne.
Miura showed that the use of $\mathrm{Ni}$ catalyst also allowed the alkynylation of azoles using terminal acetylenes. ${ }^{70}$<smiles>[R]c1c(F)c(F)cc(F)c1F</smiles>
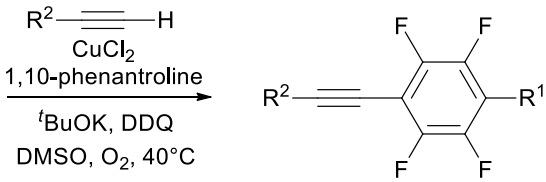

Scheme 55 Copper-catalyzed alkynylation of polyfluorobenzenes using terminal acetylenes.

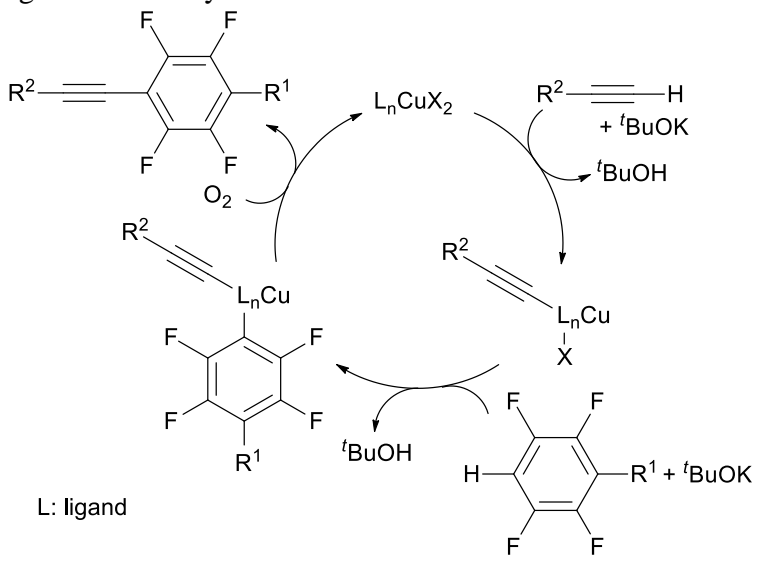

Scheme 56 Mechanism of the copper-catalyzed alkynylation of 50 polyfluorobenzenes.

$\mathrm{Li}$ and co-workers reported the $\mathrm{C} 2$ alkynylation of 1,3dimethylindole (33) using palladium catalysts (Scheme 57). ${ }^{71}$ The proposed mechanism involves $\mathrm{Pd}^{\mathrm{II}}$ acetylide formation, 55 coordination/deprotonation of the indole and then a reductive elimination. Oxygen then regenerates the Pd (II) catalyst.
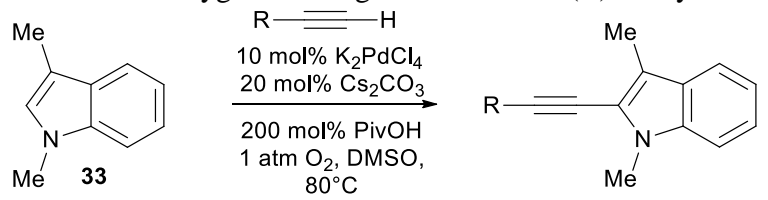

Scheme 57 Palladium-catalyzed alkynylation of 1,3dimethylindole (33) using terminal acetylenes.

60

To conclude this section, the field of direct alkynylation of aromatics has made tremendous progress in recent years. A broad variety of important heterocycles can now be alkynylated directly using a complementary approach to the classical Sonogashira 65 reaction. Nevertheless, continued advancement is needed to extend the scope of these reactions. Indeed, most of the methods are usually limited either in the scope of alkynyl substituents or in the structure of the heterocycles. More general methods are still to be discovered.

70

\subsubsection{Aliphatic C-H bonds}

The functionalization of aliphatic $\mathrm{C}-\mathrm{H}$ bonds constitutes a formidable challenge. Friedel-Craft type mechanisms are not possible in this case and $\mathrm{C}-\mathrm{H}$ activation is much more difficult. ${ }_{75}$ Developing catalytic alkynylation methods in particular asks to activate an inert $\mathrm{C}-\mathrm{H}$ bond without touching the potentially more reactive triple bond.

In 2005, Yamaguchi and co-workers reported a fascinating dialkynylation of vinyl-alkynyl and dialkynyl methane mediated by 80 gallium trichloride (Scheme 58). ${ }^{72}$ In analogy to the 
functionalization of carbonyl compounds, they proposed a mechanism involving the formation of a gallium propargylic intermediate, followed by an addition-elimination. Nevertheless, the reaction proceeded under harsh conditions and remained 5 specific to 1,4-enynes and 1,4-diynes.

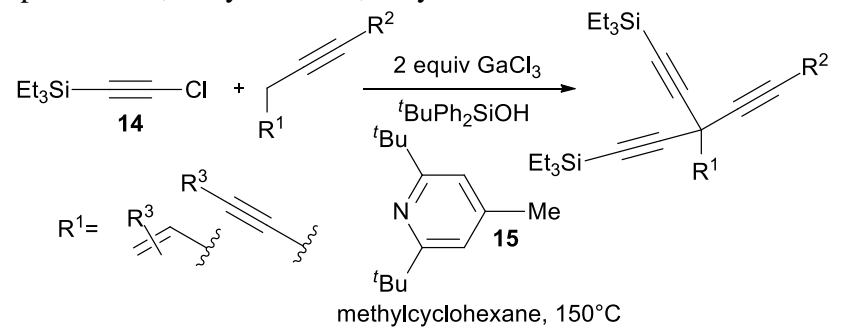

Scheme 58 1,4-enynes and 1,4-diynes bisalkynylation using chloroalkyne $\mathbf{1 4}$.

10 The first real breakthrough in the field of aliphatic $\mathrm{C}-\mathrm{H}$ alkynylation was reported by Chatani, Tobisu and Ano in 2011 (Scheme 59). ${ }^{73}$ Making use of a quinoline protecting group and the exceptional properties of trisopropylsilylbromoacetylene (26), they successfully developed the first palladium-catalyzed $\beta-\mathrm{C}-\mathrm{H}$ 15 alkynylation of acid derivatives. The reaction worked especially well for the functionalization of secondary C-H bonds and tolerated a broad range of substituents including alkyl, aryl, methoxy and trifluoromethyl groups. Even more impressive was the alkynylation of bioactive compounds, such as fatty acid ${ }_{20}$ derivative $\mathbf{3 4}, \beta$-amino acid $\mathbf{3 5}$ or a highly functionalized steroid 36. A mechanism has not yet been proposed for this new reaction, but it likely begins with $\mathrm{C}-\mathrm{H}$ activation by palladium to form a palladium alkyl intermediate. At this point, either an oxidative addition/reductive elimination or a insertion/ $\beta$-bromide 25 elimination (see Scheme 47) could be considered. Truly, these results by Chatani and co-workers constitute a milestone in the field of electrophilic alkynylation, and present a huge potential for the functionalization of bioactive building blocks, for example via Huisgen [3+2] cycloaddition with azides ("Click chemistry").
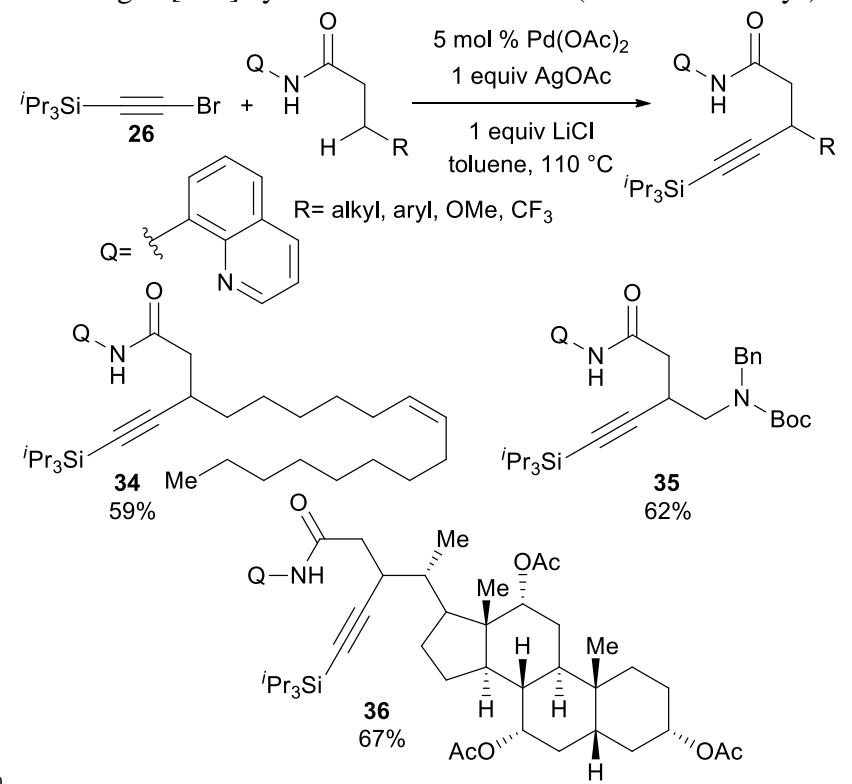

Scheme 59 Palladium-catalyzed sp3 C-H bond alkynylation using bromoalkynes.

\subsection{4 $\mathrm{C}=\mathrm{C}$ bonds}

${ }_{35}$ Olefins are broadly available building blocks, and their transformation allows a fast entry into molecular complexity, especially if one or two stereocenters are generated. Surprisingly, the alkynylation of olefins had never been achieved with electrophilic reagents, with the exception of the classic Heck 40 coupling (see section 3.2.2). However, in the Heck reaction, no new stereocenter is generated.

In 2010, Waser and co-workers introduced a new multifunctionalization of alkenes using an internal heteroatom nucleophile and an electrophilic alkynylation reagent (Scheme ${ }_{45}$ 60). Using TIPS-EBX (29) as reagent and palladium hexafluoroacetylacetonate (hfacac) as a catalyst they first developed the oxyalkynylation of electron-neutral and -deficient phenols and both aromatic and aliphatic carboxylic acids (Scheme 60, A) ${ }^{74}$ In this report, they made use of the strong 50 oxidative properties of hypervalent iodine reagents to access a $\mathrm{Pd}^{\mathrm{II}} / \mathrm{Pd}^{\mathrm{IV}}$ catalytic cycle (Scheme 61). ${ }^{75}$ An electrophilic palladium complex should allow oxypalladation of an olefin to give a more electron-rich palladium alkyl intermediate. After oxidative addition of the alkynylation reagent, reductive 55 elimination would give the elimination product. Using an in situ formed lithium palladate, the aminoalkynylation of olefins to form lactams could also be successfully developed and was applied in the synthesis of more complex indolizidine and pyrrolizidine heterocycles (Scheme $60, \mathbf{B}) .^{76}$

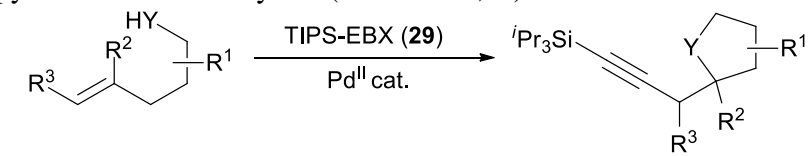

A/ Phenol and lactones: with $10 \mathrm{~mol} \% \mathrm{Pd}(\text { hfacac })_{2}, \mathrm{CH}_{2} \mathrm{Cl}_{2}$, rt

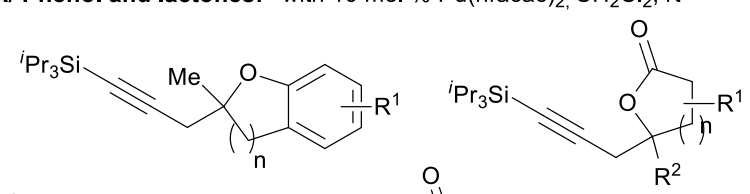

B/ Lactams:

with $10 \mathrm{~mol} \% \mathrm{PdCl}_{2}$ $\mathrm{LiCl}, \mathrm{EtOH}, \mathrm{rt}$

${ }^{60}$ Scheme 60 Palladium (II)-catalyzed oxy- and aminoalkynylation of double bonds using alkynyl benziodoxolone 29.

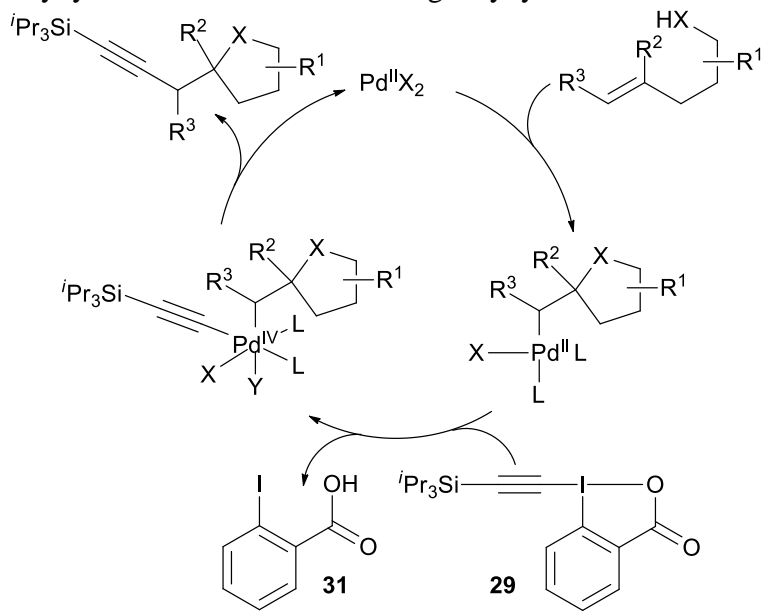

Scheme 61 Mechanism for the palladium (II)-catalyzed oxy- and 65 amino- alkynylation of double bonds. 
The $\mathrm{Pd} / \mathrm{Pd}^{\mathrm{IV}}$ protocol runs at room temperature and is tolerant to air and moisture. It also gives access to both 5- and 6membered ring. However, it could not be extended to weaker nucleophiles, such as alcohols and amides, and alkynylation was 5 possible only on primary positions. In 2011, taking inspiration from the work of Wolfe and co-workers on the related oxy- and amino-arylation reactions, ${ }^{77}$ another approach based on $\mathrm{Pd}^{0} / \mathrm{Pd}^{\mathrm{II}}$ was investigated (Scheme 62). ${ }^{78}$ This method was successful for the synthesis of both tetrahydrofurans and pyrrolidines using 10 triisopropylsilylethynyl bromide (26) and palladium DPEPhos as catalyst. Interestingly, trans-2,5-disubstituted tetrahydrofurans and cis-2,5-disubstituted pyrrolidines were obtained with high diastereoselectivity and the alkynylation of the secondary position also resulted in high yields. The first step of the catalytic 15 cycle is probably oxidative addition onto the alkynylation reagent, followed by base-mediated ligand exchange, synoxypalladation and reductive elimination (Scheme 63).

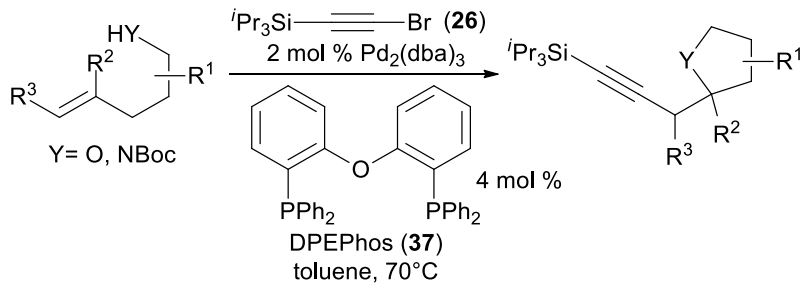

Scheme 62 Palladium (0)-catalyzed oxy- and amino20 alkynylation of double bonds using alkynyl bromide $\mathbf{2 6}$.

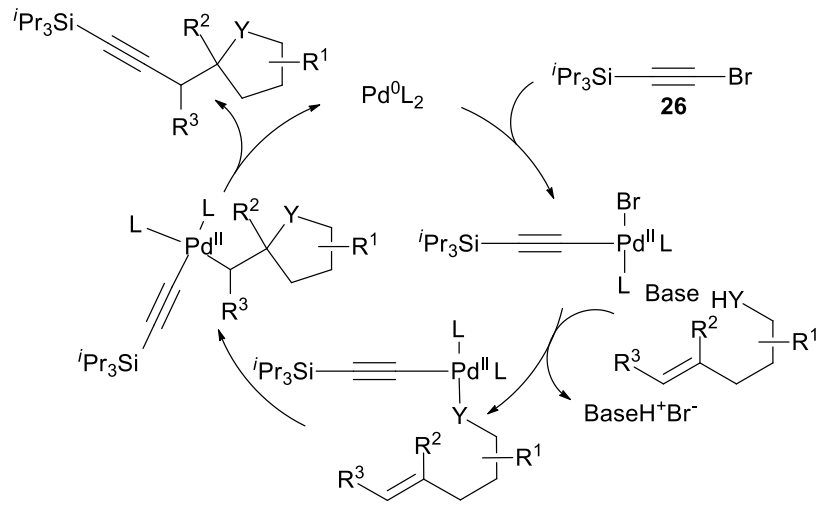

Scheme 63 Mechanism for the palladium (0)-catalyzed oxy- and amino- alkynylation of double bonds.

25

As a last example of olefin functionalization, the Pd-catalyzed reaction of bromoalkynes and norbornenes was reported by Jiang and co-workers (Scheme 64) ${ }^{79}$ In this interesting reaction, both acetylene and bromine are transferred to the olefin, which 30 resulted in the 1,3 addition product instead of the potentially expected 1,2 functionalization of the olefin. A first tentative mechanism involving oxidative addition of $\operatorname{Pd}(0)$ on the alkynyl bromide, followed by insertion, rearrangement and reductive elimination was proposed by the authors.

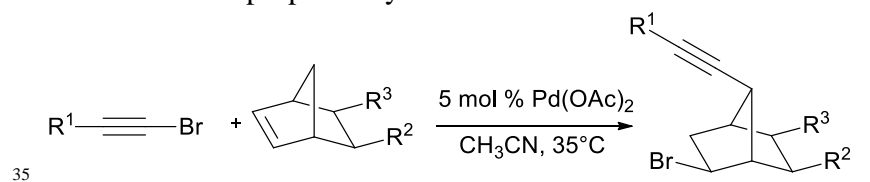

Scheme 64 Palladium-catalyzed bromoalkynylation of norbornenes using bromoalkynes.
In summary, the electrophilic alkynylation of $\mathrm{C}-\mathrm{H}$ and $\mathrm{C}=\mathrm{C}$ bond is a new and exciting area in organic chemistry. Impressive 40 results for the direct alkynylation of aromatic $\mathrm{C}-\mathrm{H}$ bonds using several different catalysts and the recent successes in Pd-catalysis for the alkynylation of aliphatic $\mathrm{C}-\mathrm{H}$ bonds and alkenes have just begun to reveal the huge potential of this approach for the efficient synthesis of acetylenes.

\section{5}

\subsection{Alkynylation of radicals}

In addition to classical nucleophiles, radical reactions represent an important complementary process. In the nineties, Fuchs and co-workers demonstrated that acetylenic triflones are excellent 50 alkyne transfer reagents for radical mediated reactions. In 1996, they showed that $\mathrm{C}-\mathrm{H}$ bonds can be efficiently alkynylated in the presence of a radical initiator (Scheme 65). ${ }^{80}$ The reaction was applied to ethers, sulfides, and cycloalkanes. Interestingly, a trifluoromethylation-alkynylation of double bonds was also 55 reported. Aromatic, alkyl and trialkylsilyl groups were tolerated on the electrophilic acetylene. ${ }^{81}$

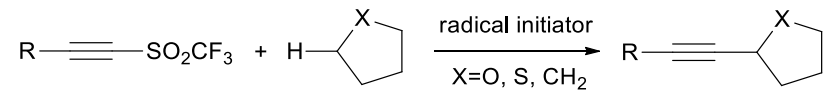

Scheme 65 Radical alkynylation of sp3 C-H bonds using alkynyl triflones.

Mechanistic investigations showed that the reaction likely proceeds via $\alpha$-attacks of the radical on the alkynyl triflones (Scheme 66) ${ }^{82} \beta$-elimination then generates the triple bond and a trifluoromethylsulfonyl radical, which then forms the highly ${ }_{65}$ reactive trifluoromethyl radical upon elimination of sulphur dioxide. Finally, the trifluoromethyl radical acts as an $\mathrm{H}$ abstractor. Nevertheless, a $\beta$-addition-rearrangement mechanism could not be fully excluded.

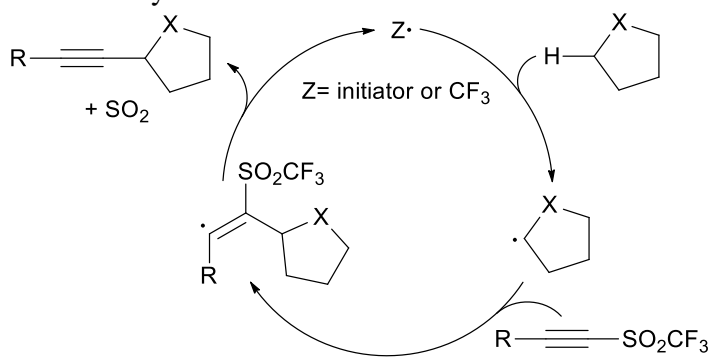

${ }_{70}$ Scheme 66 Mechanism of the radical alkynylation.

Further studies showed that alkyl halides can be alkynylated under radical conditions, leading to an efficient formal alkylation of acetylenes (Scheme 67). ${ }^{83}$ In this reaction, triisopropylsilyl 75 acetylene gave best results. This last method was orthogonal to the former one. Indeed, alkynylation of alkyl iodides can proceed in the presence of a tetrahydrofuran group in good yield.

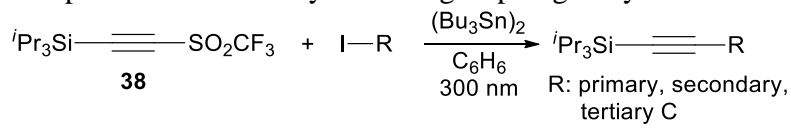

Scheme 67 Radical alkynylation of alkyl iodides.

More recently, Renaud and co-workers reported the radical alkynylation of $\beta$-alkylcatecholboranes (Scheme 68). ${ }^{84}$ Phenylsulfones were used instead of triflones. This reaction was limited to acetylenes bearing groups able to stabilize a radical at 
their $\alpha$ position, such as phenyl or trimethylsilyl. Indeed, other alkynyl phenylsulfones were known to be attacked in $\beta$-position to the sulfone by radicals. ${ }^{85}$

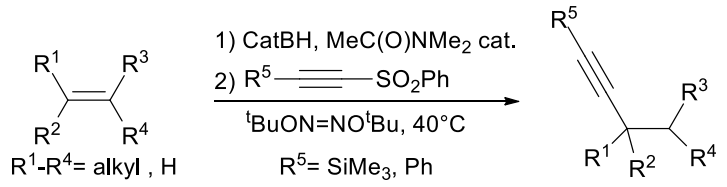

${ }_{5}$ Scheme 68 Radical alkynylation of alkyl boranes using alkynyl phenylsulfones.

In summary, alkynyl triflones and phenylsulfones are efficient reagents for the trapping of nucleophilic radicals under mild 10 conditions. Due to the large number of methods to generate radicals, they allow a broad array of alternative disconnections for the introduction of acetylene groups into organic molecules.

\section{Conclusion}

When considering disconnections to introduce acetylenes into 15 molecules, organic chemists rarely consider the possibility of an electrophilic alkynylation. Even if this is easily understandable in the context of the innate nucleophilicity of alkynes, such a limited scope restricts the flexibility of acetylene synthesis. The development and use of electrophilic triple bond synthons is 20 consequently an important task that has been partly neglected in the past. However, in recent years, impressive progress has been accomplished. For the first time, ynamides have become easily accessible, paving the way for their regular use in organic synthesis. The first enantioselective alkynylations of stabilized 25 enolates have been reported. As such, catalytic methods are now available for the alkynylation of organometallic reagents and the stereochemistry of these reactions has been investigated for the first time. Inherently more efficient methods for the direct alkynylation of $\mathrm{C}-\mathrm{H}$ and $\mathrm{C}=\mathrm{C}$ bonds have been discovered and 30 introducing acetylene on radicals is now possible. While this progress is indeed impressive, the field of electrophilic alkynylation is still in its infancy when compared to similar arylation methods. When considering the constantly increasing use of acetylenes in organic synthesis, metal catalysis, chemical 35 biology and materials science, the area is bound to remain the focus of continued research for many years to come. 


\section{Notes and references}

${ }^{a}$ Laboratory of Catalysis and Organic Synthesis, Ecole Polytechnique Fédérale de Lausanne, EPFL SB ISIC LCSO, BCH 4306, 1015 Lausanne (CH), Fax: (+) 412169397 00, E-mail: jerome.waser@epfl.ch, 5 Homepage: http://isic.epfl.ch/lcso

1 F. Diederich, P. J. Stang, R. R. Tykwinski, Acetylene Chemistry: Chemistry, Biology and Material Science; WileyVCH, Weinheim, 2005.

2 B. M. Trost and A. H. Weiss, Adv. Synth. Catal. 2009, 351, 963.

3 R. Chinchilla, C. Najera, Chem. Rev., 2007, 107, 874-922.

4 V. V.Zhdankin and P. J. Stang, Tetrahedron, 1998, 54, 1092710966.

5 In order to facilitate understanding of the key concepts in electrophilic alkynylation, we have chosen to highlight a few examples that we found particularly relevant. Of course, this review is in no means comprehensive, and many other beautiful works have also been done in the field.

6 For a recent and more general review, see: C. Liu, H. Zhang, W. Shi, A. W. Lei, Chem. Rev. 2011, 111, 1780-1824.

7 C. A. Zificsak, J. A. Mulder, R. P. Hsung, C. Rameshkumar, L.-L. Wei, Tetrahedron 2001, 57, 7575-7606.

8 G. Evano, A. Coste and K. Jouvin, Angew. Chem., Int. Ed., 2010, 49, 2840-2859.

259 P. Murch, B. L. Williamson and P. J. Stang, Synthesis, 1994, $1255-1256$

10 K. S. Feldman, M. M. Bruendl, K. Schildknegt and A. C. Bohnstedt, J. Org. Chem., 1996, 61, 5440-5452.

11 B. Witulski and T. Stengel, Angew. Chem., Int. Ed., 1998, 37, 489-492.

12 M. O. Frederick, J. A. Mulder, M. R. Tracey, R. P. Hsung, J. Huang, K. C. M. Kurtz, L. Shen and C. J. Douglas, J. Am. Chem. Soc., 2003, 125, 2368-2369.

13 J. R. Dunetz and R. L. Danheiser, Org. Lett., 2003, 5, 40114016.

14 X. Zhang, Y. Zhang, J. Huang, R. P. Hsung, K. C. M. Kurtz, J. Oppenheimer, M. E. Petersen, I. K. Sagamanova, L. Shen and M. R. Tracey, J. Org. Chem., 2006, 71, 4170-4177. And references therein.

4015 T. Hamada, X. Ye and S. S. Stahl, J. Am. Chem. Soc., 2008, 130, 833-835.

16 T. Sueda, A. Oshima and N. Teno, Org. Lett., 2011, 13, 39963999.

17 P. J. Stang, Acc. Chem. Res., 1991, 24, 304-310.

18 P. J. Stang, B. W. Surber, Z. C. Chen, K. A. Roberts, and A. G. Anderson, J. Am. Chem. Soc., 1987, 109, 228-235.

19 P. J. Stang, M. Boehshar, H. Wingert and T. Kitamura, J. Am. Chem. Soc., 1988, 110, 3272-3278.

20 P. J. Stang, T. Kitamura, M. Boehshar and H. Wingert, J. Am. Chem. Soc., 1989, 111, 2225-2230.

21 M. Ochiai, M. Kunishima, Y. Nagao, K. Fuji and E. Fujita, $J$. Chem. Soc., Chem. Comm., 1987, 1708 - 1709.

22 P. J. Stang and C. M. Crittell, J. Org. Chem., 1992, 57, 43054306.

23 Y. Gao, G. Wang, L. Chen, P. Xu, Y. Zhao, Y. Zhou and L.-B. Han, J. Am. Chem. Soc., 2009, 131, 7956-7957.

24 E. Bernoud, C. Alayrac, O. Delacroix and A.-C. Gaumont, Chem. Comm., 2011, 47, 3239-3241.

25 P. Wipf and S. Venkatraman, J. Org. Chem., 1996, 61, 80048005.

26 K. Miyamoto, Y. Nishi and M. Ochiai, Angew. Chem., Int. Ed., 2005, 44, 6896-6899.

27 F. M. Beringer and S. A. Galton, J. Org. Chem., 1965, 30, 1930-1934.

6528 M. Ochiai, M. Kunishima, Y. Nagao, K. Fuji, M. Shiro and E. Fujita, J. Am. Chem. Soc., 1986, 108, 8281-8283.

29 M. Ochiai, T. Ito, Y. Takaoka, Y. Masaki, M. Kunishima and et al., J. Chem. Soc., Chem. Commun., 1990, 118-119.

30 M. D. Bachi, N. Bar-Ner, C. M. Crittell, P. J. Stang and B. L. 140 Williamson, J. Org. Chem., 1991, 56, 3912-3915.
31 D. Fernandez Gonzalez, J. P. Brand and J. Waser, Chem. Eur. J., 2010, 16, 9457-9461

32 M. G. Moloney, J. T. Pinhey and E. G. Roche, Tetrahedron Lett., 1986, 27, 5025-5028.

33 C. J. Parkinson, T. W. Hambley and J. T. Pinhey, J. Chem. Soc., Perkin Trans. 1, 1997, 1465-1468.

34 A. S. Kende, P. Fludzinski, J. H. Hill, W. Swenson and J. Clardy, J. Am. Chem. Soc., 1984, 106, 3551-3562. And references therein.

35 T. B. Poulsen, L. Bernardi, J. Aleman, J. Overgaard and K. A. Joergensen, J. Am. Chem. Soc., 2007, 129, 441-449.

36 R. Amemiya, A. Fujii, M. Arisawa and M. Yamaguchi, $J$. Organomet. Chem., 2003, 686, 94-100.

37 Y. Nishimura, R. Amemiya and M. Yamaguchi, Tetrahedron Lett., 2006, 47, 1839-1843.

38 R. L. Smorada and W. E. Truce, J. Org. Chem, 1979, 44, 3444-3445

39 R. Sauvetre and J. F. Normant, Tetrahedron Lett., 1982, 23, 4325-4328

40 P. J. Stang and T. Kitamura, J. Am. Chem. Soc., 1987, 109, 7561-7563.

41 M. C. P. Yeh and P. Knochel, Tetrahedron Lett., 1989, 30, 4799-4802.

42 E. Hupe and P. Knochel, Angew. Chem., Int. Ed., 2001, 40, 3022-3025.

43 T. Newhouse, C. A. Lewis, K. J. Eastman and P. S. Baran, J. Am. Chem. Soc., 2010, 132, 7119-7137.

44 E. Negishi, N. Okukado, A. O. King, D. E. Van Horn and B. I. Spiegel, J. Am. Chem. Soc., 1978, 100, 2254-2256.

45 N. Miyaura, K. Yamada, H. Suginome and A. Suzuki, J. Am. Chem. Soc., 1985, 107, 972-980.

46 Y. Wen, A. Wang, H. Jiang, S. Zhu and L. Huang, Tetrahedron Lett., 2011, 52, 5736-5739.

47 S.-K. Kang, K.-Y. Jung, C.-H. Park and S.-B. Jang, Tetrahedron Lett., 1995, 36, 8047-8050.

48 G. Cahiez, O. Gager and J. Buendia, Angew. Chem., Int. Ed., 2010, 49, 1278-1281.

49 T. Thaler, P. Mayer, P. Knochel and L.-N. Guo, Angew. Chem., Int. Ed., 2011, 50, 2174-2177.

11050 W. Chodkiewicz and P. Cadiot, C.R. Hebd. Seances Acad. Sci., 1955, 241, 1055-1057.

51 For a review, see: P. Siemsen, R. C. Livingston and F. Diederich, Angew. Chem., Int. Ed., 2000, 39, 2633-2657.

52 H. Takamura, H. Wada, N. Lu and I. Kadota, Org. Lett., 2011, 13, 3644-3647.

53 W. Shi, Y. Luo, X. Luo, L. Chao, H. Zhang, A. Lei and J. Wang, J. Am. Chem. Soc., 2008, 130, 14713-14720.

54 T. N. Hoheisel and H. Frauenrath, Org. Lett., 2008, 10, 45254528.

55 K. Kobayashi, M. Arisawa and M. Yamaguchi, J. Am. Chem. Soc., 2002, 124, 8528-8529.

56 R. Amemiya, A. Fujii and M. Yamaguchi, Tetrahedron Lett., 2004, 45, 4333-4336.

57 B. A. Trofimov, Z. V. Stepanova, L. N. Sobenina, A. B. I. Mikhaleva and I. A. Ushakov, Tetrahedron Lett., 2004, 45, 6513-6516.

58 I. V. Seregin, V. Ryabova and V. Gevorgyan, J. Am. Chem. Soc., 2007, 129, 7742-7743.

59 Y. Gu and X.-M. Wang, Tetrahedron Lett., 2009, 50, 763-766.

60 M. Tobisu, Y. Ano and N. Chatani, Org. Lett., 2009, 11, 32503252 .

61 Y. Ano, M. Tobisu and N. Chatani, Org. Lett., 2012, 14, 354 357.

62 F. Besselievre and S. Piguel, Angew. Chem., Int. Ed., 2009, 48, 9553-9556.

63 N. Matsuyama, K. Hirano, T. Satoh and M. Miura, Org. Lett., 2009, 11, 4156-4159.

64 S. H. Kim and S. Chang, Org. Lett., 2010, 12, 1868-1871.

65 J. P. Brand, J. Charpentier and J. Waser, Angew. Chem. Int. Ed., 2009, 48, 9346-9349.

66 J. P. Brand and J. Waser, Org. Lett., 2012, 14, 744-747. 
67 J. P. Brand and J. Waser, Angew. Chem. Int. Ed., 2010, 49, 7304-7307.

68 T. de Haro and C. Nevado, J. Am. Chem. Soc., 2010, 132, $1512-1513$.

569 Y. Wei, H. Zhao, J. Kan, W. Su and M. Hong, J. Am. Chem. Soc., 2010, 132, 2522-2523.

70 N. Matsuyama, M. Kitahara, K. Hirano, T. Satoh and M. Miura, Org. Lett., 2010, 12, 2358-2361.

71 L. Yang, L. Zhao and C.-J. Li, Chem. Comm., 2010, 46, 41844186.

72 R. Amemiya, K. Suwa, J. Toriyama, Y. Nishimura and M. Yamaguchi, J. Am. Chem. Soc., 2005, 127, 8252-8253.

73 Y. Ano, M. Tobisu and N. Chatani, J. Am. Chem. Soc., 2011, 133, 12984-12986.

1574 S. Nicolai, S. Erard, D. F. Gonzalez and J. Waser, Org. Lett., 2010, 12, 384-387.

75 For a review on $\mathrm{Pd}^{\mathrm{II}} / \mathrm{Pd}^{\mathrm{IV}}$ catalysis, see: K. Muniz, Angew. Chem., Int. Ed., 2009, 48, 9412-9423

20

76 S. Nicolai, C. Piemontesi and J. Waser, Angew. Chem., Int. Ed., 2011, 50, 4680-4683.

77 J. P. Wolfe, Synlett, 2008, 2913-2937. And references therein.

78 S. Nicolai and J. Waser, Org. Lett., 2011, 13, 6324-6327.

79 Y. Li, X. Liu, H. Jiang, B. Liu, Z. Chen and P. Zhou, Angew. Chem., Int. Ed., 2011, 50, 6341-6345.

2580 J. Gong and P. L. Fuchs, J. Am. Chem. Soc., 1996, 118, 44864487.

81 J. Xiang, W. Jiang and P. L. Fuchs, Tetrahedron Lett., 1997, 38, 6635-6638.

82 J. S. Xiang and P. L. Fuchs, Tetrahedron Lett., 1996, 37, 5269-5272.

83 J. Xiang and P. L. Fuchs, Tetrahedron Lett., 1998, 39, 85978600.

84 A.-P. Schaffner, V. Darmency and P. Renaud, Angew. Chem., Int. Ed., 2006, 45, 5847-5849.

3585 Review on acetylinic sulphones: B. Thomas G, Tetrahedron, 2001, 57, 5263-5301. 


\section{Journal Name}

Cite this: DOI: $10.1039 / \mathrm{c0xx00000x}$

www.rsc.org/xxxxxx

\section{ARTICLE TYPE}

\section{Graphical Abstract}

This tutorial review reveals the tremendous, but often neglected, potential of electrophilic alkynylation methods for the synthesis of acetylenes.

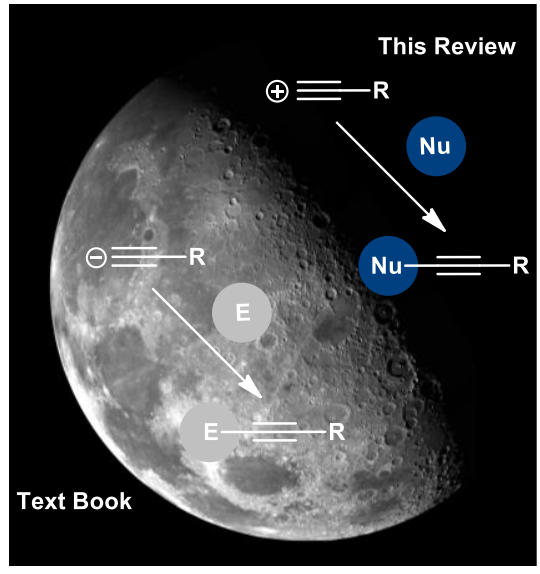

